PREPRINT of J. Math. Phys. 56 (2015), 103513

\title{
Pseudospectra in non-Hermitian quantum mechanics
}

\author{
D. Krejčiřík ${ }^{a}$, P. Siegl ${ }^{a, b}$, M. Tater ${ }^{a}$ and J. Viola ${ }^{c}$ \\ a) Department of Theoretical Physics, Nuclear Physics Institute ASCR, \\ 25068 Ře ž, Czech Republic. \\ b) Mathematical Institute, University of Bern, Alpeneggstrasse 22, 3012 \\ Bern, Switzerland. \\ c) Département de Mathématiques, Université de Nantes, 2 rue de la \\ Houssinière, BP 92208, 44322 Nantes Cedex 3, France.
}

12th October 2015

\begin{abstract}
We propose giving the mathematical concept of the pseudospectrum a central role in quantum mechanics with non-Hermitian operators. We relate pseudospectral properties to quasi-Hermiticity, similarity to selfadjoint operators, and basis properties of eigenfunctions. The abstract results are illustrated by unexpected wild properties of operators familiar from $\mathcal{P T}$-symmetric quantum mechanics.
\end{abstract}

Mathematics Subject Classification (2010):

Primary: 47A10, 81Q12; Secondary: 34L10, 35A27, 47B44.

Keywords: quantum mechanics with non-self-adjoint operators, pseudospectra, semiclassical pseudomodes, microlocal analysis, JWKB approximation, similarity to self-adjoint operators, quasi-Hermiticity, metric operator, $\mathcal{P} \mathcal{T}$-symmetry, imaginary Airy operator, imaginary cubic oscillator, complex rotated and shifted harmonic oscillators, elliptic quadratic operators, wild basis properties, spectral instability

\section{Introduction}

In the highly non-normal case, vivid though the image may be, the location of the eigenvalues may be as fragile an indicator of underlying character as the hair colour of a Hollywood actor.

Trefethen and Embree, [77, p. 11]

It has long been known to numerical analysts that many important spectral properties of self-adjoint operators are lost when considering non-normal operators. In the 2005 monograph 77, Trefethen and Embree discuss decades of ongoing research and advocate the use of pseudospectra instead of spectra when studying a matrix or operator which is non-self-adjoint or non-normal. 
In this paper we stress the importance of pseudospectra, a set which measures the instability of the spectrum of a non-normal operator, in the so-called "non-Hermitian quantum mechanics". Contrary to a common misconception, we demonstrate that the spectrum alone contains by far insufficient information to draw any quantum-mechanically relevant conclusions for non-Hermitian operators. In particular, the fact that the spectrum of an operator is real, or even the presence of a reduction to a self-adjoint operator using an unbounded similarity transformation, is not sufficient to guarantee that an operator with non-trivial pseudospectrum has a meaning in the context of conventional quantum mechanics.

By non-Hermitian quantum mechanics we mean the attempts of Bender et al. [15, 16, to extend quantum mechanics to include observables represented by $\mathcal{P} \mathcal{T}$-symmetric non-Hermitian operators, see [1], with real spectra. Here, $\mathcal{P} \mathcal{T}$ symmetry refers to the invariance of an operator $H$ on the Hilbert space $L^{2}\left(\mathbb{R}^{d}\right)$ with respect to a simultaneous parity and time reversal, i.e.,

$$
[H, \mathcal{P T}]=0,
$$

where $(\mathcal{P} \psi)(x):=\psi(-x)$ and $(\mathcal{T} \psi)(x):=\overline{\psi(x)}$. It has been argued that if the operator possesses, in addition to the obvious $\mathcal{P} \mathcal{T}$-symmetry, a special hidden symmetry - a so-called $\mathcal{C}$-symmetry - then indeed the spectrum of $H$ is real. It has furthermore been suggested that a consistent quantum theory can be built by changing the inner product into one for which the operator $H$ is Hermitian and the time evolution is unitary. The procedure can be understood by the concept of pseudo-Hermiticity as developed by Mostafazadeh [52, 53, 54: a CPTsymmetric operator can be transformed into a self-adjoint operator $H_{\mathrm{sa}}=H_{\mathrm{sa}}^{*}$ via a similarity transformation $\Omega$, i.e.,

$$
H_{\mathrm{sa}}=\Omega H \Omega^{-1} .
$$

The latter is the basis for a possible quantum-mechanical interpretation of $H$ as an equivalent representation of a physical observable which would be conventionally represented by the self-adjoint operator $H_{\mathrm{sa}}$.

If $\Omega$ in (2) is bounded and boundedly invertible, $c f$. [2, although not necessarily unitary, then indeed the spectra of $H_{\text {sa }}$ and $H$ coincide and the pseudospectra are related by simple approximate inclusions; see (8). Moreover, if the spectrum is discrete, the eigenfunctions of $H$ and $H_{\mathrm{sa}}$ share essential basis properties. In this case, the $\mathcal{P} \mathcal{T}$-symmetry can be understood through an older notion of quasi-Hermiticity [30, 66] and the quantum-mechanical description of $H$ via $H_{\mathrm{sa}}$ is consistent: $H$ and $H_{\mathrm{sa}}$ represent the same physical system.

However, problems arise if $\Omega$ or $\Omega^{-1}$ entering the fundamental relation (2) are allowed to be unbounded. We list several potential pitfalls below. We do not claim that there are no physical problems where an unbounded similarity transformation could be useful (in fact, there are!), nevertheless, if any of the pathological situations described below occur, $H_{\mathrm{sa}}$ and $H$ cannot be viewed as equivalent representatives of the same physical observable in quantum mechanics.

1. It is not always easy to give a good meaning to the operator identity (2) when taking into account the respective domains. The relation (2) may hold on some particular functions, e.g. $C_{0}^{\infty}\left(\mathbb{R}^{d}\right)$, but the operator identity may not be satisfied. 
2. Spectra are not preserved by unbounded transformations. It may well happen that the spectrum of $H$ is purely discrete, while $H_{\mathrm{sa}}$ has no eigenvalues or some continuous spectrum, and vice versa.

3. Unbounded transformations may turn a nice (even orthonormal) eigenbasis to a set of functions that cannot form any kind of reasonable basis.

4. Spectra of non-Hermitian operators are known to lie deep inside very large pseudospectra, while the pseudospectrum of a self-adjoint operator is just a tubular neighbourhood of its spectrum. Consequently, the spectrum of $H_{\mathrm{sa}}$ is stable under small perturbations, while an arbitrarily small perturbation of $H$ can create eigenvalues very far from the spectrum of $H$.

5. Even if the spectrum of $H$ were purely real, $-i H$ does not need to be the generator of a bounded semigroup. In fact, a wild behaviour of the pseudospectrum of $H$ prevents to associate a bounded time-evolution to $H$ via the Schrödinger equation ( $c f$. [26, Thm. 8.2.1]).

The objective of this paper is to demonstrate by a careful mathematical analysis that these commonly overlooked problems do appear in more or less famous models of non-Hermitian quantum mechanics and to emphasize that the concept of pseudospectra gives important information missing in prior works. In conclusion, the present study necessarily casts doubt on certain commonly accepted conclusions based on formal manipulations in the physical literature, cf. the reviews [14, 55, particularly on the physical relevance of $\mathcal{P} \mathcal{P}$-symmetry in the quantum-mechanical context. We notably refer to the concrete examples presented in Section 7 for specific controversies. We also remark that the unbounded time-evolution has been recently studied more explicitly for some of the models presented below, namely in 36 for the gauged oscillator (see Section 7.7) and in [8, 9, for quadratic operators (see Section 7.8).

Our approach relies on standard tools of modern functional analysis, nonetheless, several innovations appear. Particularly, we construct new pseudomodes for the (non-semiclassical) shifted harmonic oscillator, where the scaling leads to the fractional power of $h, c f$. Theorem 7 We also give a new and very short proof of the rate of spectral projection growth for the rotated oscillator analysed previously in [27, 38, 79], cf. Appendix B. Last but not least, we establish an explicit unitary equivalence between the rotated and gauged oscillator (Swanson's model), which has not been noticed previously. We furthermore describe how to identify an equivalent rotated oscillator for each such quadratic operator in a class identified in 58 .

The paper is organized as follows. In the Section 2 we summarize some basic properties of pseudospectra. Since $\mathcal{P} \mathcal{T}_{\text {-symmetry is a special example of an }}$ antilinear symmetry, we briefly discuss pseudospectral properties of operators commuting with antiunitary operators in Section 3, Our main mathematical tool for proving the existence of large pseudospectra is stated as Theorem 1 of Section 4 it is based on a construction of pseudomodes of semiclassical operators adapted from [29. In Section [5 we relate the pseudospectrum to the concept of quasi-Hermiticity and similarity to a self-adjoint operator. A relationship between basis properties of eigenfunctions is pointed out in Section 6 . Finally, in Section 7 (which occupies the bulk of the paper), we present a number of non-self-adjoint ordinary differential operators exhibiting striking spectral 
and pseudospectral properties; they will serve as an illustration of the abstract operator-theoretic methods summarized in this paper. Certain technical proofs are reserved for the Appendix.

\section{Pseudospectra}

The notion of pseudospectra arose as a result of the realization that several pathological properties of highly non-Hermitian operators were closely related. We refer to by now classical monographs by Trefethen and Embree [77] and Davies 26 for more information on the subject and many references.

Let $H$ be a closed densely defined operator (bounded or unbounded) on a complex Hilbert space $\mathcal{H}$. The spectrum of $H$, denoted by $\sigma(H)$, consists of those complex points $z$ for which the resolvent $(H-z)^{-1}$ does not exist as a bounded operator on $\mathcal{H}$. If $\mathcal{H}$ were finite-dimensional, then the spectrum of $H$ would be exhausted by eigenvalues (i.e. those complex numbers $\lambda$ for which $H-\lambda$ is not injective). In general, however, there are additional parts of the spectrum composed of those $\lambda$ which are not eigenvalues but for which $H-\lambda$ : $\operatorname{Dom}(H) \rightarrow \mathcal{H}$ is not bijective: depending on whether the range $\operatorname{Ran}(H-\lambda)$ is dense in $\mathcal{H}$ or not, one speaks about the continuous or residual spectrum, respectively.

The complement of $\sigma(H)$ in $\mathbb{C}$ is called the resolvent set of $H$. The numerical range $\operatorname{Num}(H)$ of $H$ is defined by the set of all complex numbers $(\psi, H \psi)$ where $\psi$ ranges over all $\psi$ from the operator domain $\operatorname{Dom}(H)$ with $\|\psi\|=1$.

Given a positive number $\varepsilon$, we define the $\varepsilon$-pseudospectrum (or simply pseudospectrum) of $H$ as

$$
\sigma_{\varepsilon}(H):=\sigma(H) \cup\left\{z \in \mathbb{C} \mid\left\|(H-z)^{-1}\right\|>\varepsilon^{-1}\right\} ;
$$

sometimes the convention that $\left\|(H-z)^{-1}\right\|=\infty$ for $z \in \sigma(H)$ is used. (We refer to the interesting essays 67, 68, by Shargorodsky on the distinction between the definition of pseudospectra with strict and non-strict inequalities.) Here we summarize some basic well-known properties of pseudospectra.

- Topology. For every $\varepsilon>0, \sigma_{\varepsilon}(H)$ is a non-empty open subset of $\mathbb{C}$ and any bounded connected component of $\sigma_{\varepsilon}(H)$ has a non-empty intersection with $\sigma(H)$. (If the spectrum of $H$ is empty, then $\sigma_{\varepsilon}(H)$ is unbounded for every $\varepsilon>0$.) These facts follow from the subharmonicity of $\left\|(H-z)^{-1}\right\|$ as a function of $z \notin \sigma(H)$ to $(0, \infty)$.

- Relation to spectra. The pseudospectrum always contains an $\varepsilon$-neighbourhood of the spectrum, and if $\mathbb{C} \backslash \overline{\operatorname{Num}(H)}$ is connected and has a non-empty intersection with the resolvent set of $H$, the pseudospectrum is in turn contained in an $\varepsilon$-neighbourhood of the numerical range:

$$
\{z \in \mathbb{C} \mid \operatorname{dist}(z, \sigma(H))<\varepsilon\} \subseteq \sigma_{\varepsilon}(H) \subseteq\{z \in \mathbb{C} \mid \operatorname{dist}(z, \overline{\operatorname{Num}(H)})<\varepsilon\} .
$$

The first inclusion follows from the bound $\left\|(H-z)^{-1}\right\| \geq \operatorname{dist}(z, \sigma(H))^{-1}$, which is valid for any operator. Since equality holds there if $H$ is self-adjoint (or more generally normal, i.e. $H^{*} H=H H^{*}$ ), it follows that the pseudospectra for such 
operators give no additional information not already given by the spectrum. On the other hand, if $H$ is "highly non-self-adjoint", the pseudospectrum $\sigma_{\varepsilon}(H)$ is typically "much larger" than the $\varepsilon$-neighbourhood of the spectrum. In any case, the second inclusion shows that the pseudospectra are well behaved outside the numerical range.

- Spectral instability. There is an important property (known sometimes as the Roch-Silberman theorem 64, although the result is already mentioned in 63.) relating the pseudospectra to the stability of the spectrum under small perturbations:

$$
\sigma_{\varepsilon}(H)=\bigcup_{\|V\|<\varepsilon} \sigma(H+V)
$$

The importance of this property is summarized by the following statement from [25]:

Very large pseudospectra are always associated with eigenvalues which are very unstable with respect to perturbations. This is clearly of great importance to numerical analysts: if a spectral problem is unstable enough, no numerical procedure can enable one to find the eigenvalues, whose significance therefore becomes a moot point.

The relation (5) likely lends the strongest support for the usage of pseudospectra instead of spectra in the case of non-Hermitian operators.

- Pseudomodes. A complex number $z$ belongs to $\sigma_{\varepsilon}(H)$ if, and only if, $z \in$ $\sigma(H)$ or $z$ is a pseudoeigenvalue (or approximate eigenvalue), i.e.,

$$
\|(H-z) \psi\|<\varepsilon\|\psi\| \quad \text { for some } \quad \psi \in \operatorname{Dom}(H) .
$$

Any $\psi$ satisfying (6) is called a pseudoeigenvector (or pseudoeigenfunction or pseudomode). Again, for operators $H$ which are far from self-adjoint, pseudoeigenvalues may not be close to the spectrum of $H$. This is particularly striking if we realize that these pseudoeigenvalues can be turned into true eigenvalues by a very small perturbation, $c f$. (5). What is more, we can often construct very nice (e.g. smooth and with compact support) pseudoeigenfunctions; see Section 4 and the references therein.

- Adjoints. Using the identity $\left\|\left(H^{*}-\bar{z}\right)^{-1}\right\|=\left\|(H-z)^{-1}\right\|$, it is easy to see that the pseudospectrum of $H^{*}$ is given by the mirror image of $\sigma_{\varepsilon}(H)$ with respect to the real axis, i.e.,

$$
\lambda \in \sigma_{\varepsilon}(H) \Longleftrightarrow \bar{\lambda} \in \sigma_{\varepsilon}\left(H^{*}\right) .
$$

- Similarity. Let the similarity relation (2) hold with a bounded and boundedly invertible operator $\Omega$. Then the operators $H$ and $H_{\text {sa }}$ have the same spectra, but their pseudospectra may be very different, unless the condition number $\kappa:=\|\Omega\|\left\|\Omega^{-1}\right\| \geq 1$ is fairly close to one. Indeed, we have

$$
\sigma_{\varepsilon / \kappa}(H) \subseteq \sigma_{\varepsilon}\left(H_{\mathrm{sa}}\right) \subseteq \sigma_{\varepsilon \kappa}(H)
$$




\section{Antilinear symmetry}

We understand $\mathcal{P} \mathcal{T}$-symmetry as a special example of invariance of a closed densely defined operator $H$ with respect to an antiunitary transformation $\mathcal{S}$, i.e.,

$$
[H, \mathcal{S}]=0 .
$$

Recall that $\mathcal{S}$ is an antiunitary transformation if $\mathcal{S}$ is a bijective antilinear operator on $\mathcal{H}$ satisfying $(\mathcal{S} \phi, \mathcal{S} \psi)=(\psi, \phi)$ for every $\phi, \psi \in \mathcal{H}$. As usual for the commutativity of an unbounded operator and a bounded operator [44, Sec. III.5.6], we understand (9) by the operator relation $\delta H \subseteq H \mathcal{S}$. In other words, whenever $\psi \in \operatorname{Dom}(H)$, the image $\mathcal{S} \psi$ also belongs to $\operatorname{Dom}(H)$ and $\mathcal{S} H \psi=H \mathcal{S} \psi$.

- Symmetry. It is well known that the spectra of $\mathcal{P} \mathcal{T}$-symmetric operators on $L^{2}\left(\mathbb{R}^{d}\right)$ are symmetric with respect to the real axis. In our general situation (9), this follows from the identity

$$
(H-z)^{-1}=\mathcal{S}^{-1}(H-\bar{z})^{-1} \mathcal{S},
$$

which is valid for any $z$ in the resolvent set of $H$; the symmetry can then be deduced from (9). Furthermore, (10) yields the same relation for the pseudospectra of $\mathcal{S}$-symmetric operators $H$ :

$$
\lambda \in \sigma_{\varepsilon}(H) \Longleftrightarrow \bar{\lambda} \in \sigma_{\varepsilon}(H) .
$$

This identity holds trivially when the resolvent set of $H$ is empty.

- $\mathcal{J}$-self-adjointness. An alternative framework for $\mathcal{P} \mathcal{P}$-symmetric operators was suggested in [19] in terms of $\mathcal{J}$-self-adjoint operators. Here $\mathcal{J}$ is a conjugation, i.e. an antiunitary involution, and $H$ is said $\mathcal{J}$-self-adjoint if $H^{*}=\mathcal{J} H \mathcal{J}$, cf. [33, Sec. III.5]. (The present $\mathcal{J}$-self-adjointness should not be confused with a terminologically similar but different concept in Krein spaces [7, 37.) An example of such a $\mathcal{J}$ on $L^{2}\left(\mathbb{R}^{d}\right)$ is $\mathcal{T}$, i.e. complex conjugation. A particularly useful property of $\mathcal{J}$-self-adjoint operators is that their residual spectrum is always empty, $c f .[19$.

\section{Microlocal analysis}

It was Davies 23] who first realized that and how semiclassical methods can be applied to the study of pseudospectra of non-Hermitian Schrödinger operators. Shortly thereafter, Zworski [81 pointed out that Davies' discoveries could be related to long-established results in the microlocal theory of partial differential operators due to Hörmander and others. We refer to a paper of Dencker, Sjöstrand and Zworski 29] for an important development of the idea in the context of pseudodifferential operators. We state here a version of their general result for the special case of differential operators with analytic coefficients in one dimension, in a formulation given in [77, Thm. 11.1].

We recall some standard notions first. Let $h>0$ be a (small) parameter. Given continuous functions $a_{j}: \mathbb{R} \rightarrow \mathbb{C}$, with $j=0, \ldots, n$, we define a symbol

$$
f(x, \xi):=\sum_{j=0}^{n} a_{j}(x)(-i \xi)^{j}, \quad(x, \xi) \in \mathbb{R}^{2},
$$


and the associated semiclassical differential operator

$$
H_{h}:=\sum_{j=0}^{n} a_{j}(x) h^{j} \frac{\mathrm{d}^{j}}{\mathrm{~d} x^{j}}, \quad \operatorname{Dom}\left(H_{h}\right):=C_{0}^{\infty}(\mathbb{R}) .
$$

The Poisson bracket $\{\cdot, \cdot\}$ is defined as

$$
\{u, v\}:=\frac{\partial u}{\partial \xi} \frac{\partial v}{\partial x}-\frac{\partial u}{\partial x} \frac{\partial v}{\partial \xi}
$$

and, for $u=f, v=\bar{f}$, it simplifies to

$$
\{f, \bar{f}\}=2 i\left(\frac{\partial \operatorname{Im} f}{\partial \xi} \frac{\partial \operatorname{Re} f}{\partial x}-\frac{\partial \operatorname{Re} f}{\partial \xi} \frac{\partial \operatorname{Im} f}{\partial x}\right) .
$$

The closure of the set

$$
\Lambda:=\left\{f(x, \xi):(x, \xi) \in \mathbb{R}^{2}, \frac{1}{2 i}\{f, \bar{f}\}(x, \xi)>0\right\}
$$

is referred to as the semiclassical pseudospectrum of $H_{h}, c f$. 29. In the special case of $H_{h}$ being a Schrödinger operator with an analytic potential, the condition $\frac{1}{2 i}\{f, \bar{f}\}(x, \xi)>0$ reduces to $\operatorname{Im} V^{\prime}(x) \neq 0$ and $\xi \neq 0$, because the sign of $\xi$ can be chosen freely, and it is also equivalent to the twist condition of [77, Sec. III.11]. The nonvanishing of $\{f, \bar{f}\}$ is a classical analogue of the operator $H_{h}$ not being normal 29.

Theorem 1 (Semiclassical pseudomodes.). Let the functions $a_{j}, j=0, \ldots, n$, be analytic and let $H_{h}$ be the semiclassical differential operator (13). Then for any $z \in \Lambda$, there exist $C=C(z)>1, h_{0}=h_{0}(z)>0$ and an $h$-dependent family of $C_{0}^{\infty}(\mathbb{R})$ functions $\left\{\psi_{h}\right\}_{0<h \leq h_{0}}$ with the property that, for all $0<h \leq h_{0}$,

$$
\left\|\left(H_{h}-z\right) \psi_{h}\right\|<C^{-1 / h}\left\|\psi_{h}\right\| .
$$

Such of family of functions is called a pseudoeigenfunction (or pseudomode) for the operator $H_{h}$ corresponding to pseudoeigenvalue (or approximate eigenvalue) $z$.

In Appendix $\mathrm{A}$, we include a proof of the theorem in the special case of Schrödinger operators.

The theorem can be generalized significantly beyond the restrictive assumption that the coefficients are globally analytic. As stated in [77, Thm. 11.1], one only needs that the $a_{j}$ are analytic in a neighborhood of some $x_{0} \in \mathbb{R}$ corresponding to an $\left(x_{0}, \xi_{0}\right) \in \mathbb{R}^{2}$ putting $z \in \Lambda$. Furthermore, 29] shows the existence of pseudomodes for pseudodifferential operators (which include differential operators) whose symbols are only assumed to be smooth (and bounded). The price of abandoning the assumption of analytic coefficients is a slower rate of growth. In the smooth case, instead of an upper bound of $C^{-1 / h}\left\|\psi_{h}\right\|$ one has an upper bound for each $N \in \mathbb{N}$ and constant $C(N)>0$ depending on $N$ :

$$
\left\|\left(H_{h}-z\right) \psi_{h}\right\|<\frac{h^{N}}{C(N)}\left\|\psi_{h}\right\|,
$$

for all $0<h \leq h_{0}$. For the examples to follow, it will be sufficient to consider the analytic case as written in Theorem 1 
Although Theorem 1 is stated for semiclassical operators, scaling techniques allow its application to non-semiclassical operators where the spectral parameter tends to infinity. This is based on the principle that the semiclassical limit is equivalent to the high-energy limit after a change of variables; this principle is made concrete in many of the examples below. For further details, the reader could consult 82 .

\section{$5 \quad$ Metric operators}

The similarity relation (2) is closely related to the quasi-Hermiticity of $H$ :

$$
H^{*} \Theta=\Theta H,
$$

where $\Theta$ is a positive operator called a metric 30 , 66. The terminology comes from the observation that $H$ is formally self-adjoint with respect to the modified inner product $\langle\cdot, \Theta \cdot\rangle$. More precisely, we have

Proposition 2. The operator $H$ is similar to a self-adjoint operator via a bounded and boundedly invertible positive transformation (i.e. (2) holds) if, and only if, $H$ is quasi-Hermitian with a positive, bounded, and boundedly invertible metric (i.e. (16) holds).

Proof. If $H$ satisfies (16) with a bounded and boundedly invertible $\Theta>0$, then the similarity relation (2) to a self-adjoint operator $h$ holds with any $\Omega$ satisfying the decomposition $\Theta=\Omega^{*} \Omega$. Conversely, if (2) holds with a bounded and boundedly invertible $\Omega$, then it is easy to check that (16) holds with $\Theta=$ $\Omega^{*} \Omega>0$.

As emphasized in [71, fundamental problems arise if one starts to relax the conditions on boundedness or bounded invertibility of the transformations.

- Trivial pseudospectra. We say that the pseudospectrum of $H$ is trivial if there exists a fixed constant $C$ such that, for all $\varepsilon>0$,

$$
\sigma_{\varepsilon}(H) \subseteq\{z \in \mathbb{C} \mid \operatorname{dist}(z, \sigma(H))<C \varepsilon\} .
$$

That is, the pseudospectrum of $H$ is contained in a tubular neighbourhood of the spectrum of $H$ (although of possibly larger radius than $\varepsilon$ ). Recalling the text below (4), the pseudospectra of self-adjoint and normal operators are trivial.

Proposition 3. Let $H$ be quasi-Hermitian (16) with a positive, bounded and boundedly invertible metric. Then the pseudospectrum of $H$ is trivial.

Proof. It is enough to recall Proposition 2 and (8); the condition number plays the role of the constant $C$.

Proposition 3 can be conveniently used in the reverse sense, where the presence of nontrivial pseudospectrum for a given operator $H$ immediately implies that the operator cannot possess a physically relevant (i.e. bounded and boundedly invertible) metric. 


\section{Basis properties}

Let $H$ be an operator with compact resolvent throughout this section. Then the spectrum of $H$ consists entirely of isolated eigenvalues with finite (algebraic) multiplicities. Below, we recall that similarity to a self-adjoint operator, or quasi-Hermiticity, is equivalent to having the set of eigenvectors form a Riesz basis.

First, we recall the definition of a basis. We say that $\left\{\psi_{k}\right\}_{k=1}^{\infty}$ is a (Schauder or conditional) basis if every $\psi \in \mathcal{H}$ has a unique expansion in the vectors $\left\{\psi_{k}\right\}$, i.e.

$$
\forall \psi \in \mathcal{H}, \quad \exists !\left\{\alpha_{k}\right\}_{k=1}^{\infty}, \quad \psi=\sum_{k=1}^{\infty} \alpha_{k} \psi_{k},
$$

where the infinite sum is understood as a limit in the strong topology of $\mathcal{H}$.

- Riesz basis. We say that $\left\{\psi_{k}\right\}_{k=1}^{\infty}$, normalized to 1 in $\mathcal{H}$, forms a Riesz (or unconditional) basis if it forms a basis and the inequality

$$
\forall \psi \in \mathcal{H}, \quad C^{-1}\|\psi\|^{2} \leq \sum_{k=1}^{\infty}\left|\left\langle\psi_{k}, \psi\right\rangle\right|^{2} \leq C\|\psi\|^{2}
$$

holds with a positive constant $C$ independent of $\psi$ (see [26, Thm.3.4.5] for equivalent formulations). In view of the Parseval equality, for any self-adjoint operator with a purely discrete spectrum one may choose orthonormal eigenvectors which form a Riesz basis with $C=1$. For non-self-adjoint operators, however, it is not even clear that the eigenfunctions form a basis or even a complete set in $\mathcal{H}$.

Proposition 4. Let $H$ be an operator with compact resolvent for which $\sigma(H) \subset$ $\mathbb{R}$. Then $H$ is quasi-Hermitian with a positive, bounded and boundedly invertible metric (i.e. (16) holds) if, and only if, the eigenfunctions of $H$ form a Riesz basis (i.e. (18) holds).

Proof. If $H$ is quasi-Hermitian with bounded, boundedly invertible, and positive metric $\Theta$, then, by Proposition 2, $H$ is similar to a self-adjoint operator $h$ via a bounded and boundedly invertible transformation $\Omega$. Consequently, $\Omega \psi_{k} /\left\|\Omega \psi_{k}\right\|$ form a complete orthonormal family in $\mathcal{H}$ satisfying the Parseval inequality, from which (18) follows. Conversely, assuming (18), we construct a bounded and boundedly invertible positive operator $L:=\sum_{k=1}^{\infty} \psi_{k}\left\langle\psi_{k}, \cdot\right\rangle$. It is easy to check that (16) holds with $\Theta=L^{-1}$.

Combining Proposition 4 with Proposition 3 , we see that the pseudospectrum can be employed as a useful indicator of whether a non-self-adjoint operator possesses a Riesz basis.

- No basis. Eigensystems of non-self-adjoint operators can have very wild basis properties. We recall that if $\left\{\psi_{k}\right\}_{k=1}^{\infty}$ is a basis, then there exists a sequence $\left\{\phi_{k}\right\}_{k=1}^{\infty}$ for which the pair $\left\{\psi_{k}\right\},\left\{\phi_{k}\right\}$ is biorthogonal, i.e. $\left\langle\phi_{m}, \psi_{n}\right\rangle=\delta_{m, n}$, such that $\alpha_{k}=\left\langle\phi_{k}, \psi\right\rangle$, cf. [26, Lem. 3.3.1]. Let us denote the associated onedimensional projections as

$$
P_{k}:=\psi_{k}\left\langle\phi_{k}, \cdot\right\rangle
$$


The uniform boundedness principle is used to derive the following standard result.

Proposition 5. If $\left\{\psi_{k}\right\}_{k=1}^{\infty}$ is a basis, then both $P_{k}$ and $\sum_{k=1}^{N} P_{k}$ are uniformly bounded in $\mathcal{H}$.

Proof. The definition (17) implies that, for every $\psi \in \mathcal{H}$,

$$
\forall \varepsilon>0, \exists N_{\psi, \varepsilon}, \forall n, m>N_{\psi, \varepsilon}, \quad\left\|\sum_{k=1}^{n} P_{k} \psi-\sum_{k=1}^{m} P_{k} \psi\right\|<\varepsilon .
$$

In particular, putting $m:=n-1$ and $\varepsilon:=1$, we obtain $\left\|P_{n} \psi\right\|<1$ if $n>$ $N_{\psi, \varepsilon}$. Consequently, $\sup _{k}\left\|P_{k} \psi\right\|<\infty$ for every $\psi \in \mathcal{H}$. Finally, the uniform boundedness principle [61, Thm. III.9] yields $\sup _{k}\left\|P_{k}\right\|<\infty$. The proof of the second claim is analogous, see also [26, Lem. 3.3.3].

For operators $H$ with positive discrete spectrum, the basis property of the eigensystem of $H$ may be excluded by the following corollary of [24, Thm. 3].

Proposition 6. Let $H$ be an operator with compact resolvent, let its eigenvalues be simple and satisfy $\lambda_{n} \geq b n^{\beta}$ for some $b, \beta>0$ and all $n \in \mathbb{N}$ and let the corresponding eigenvectors form a basis. Then there exist positive constants $k, m$ such that

$$
\left\|(H-z)^{-1}\right\| \leq k \frac{\left(1+|z|^{2}\right)^{\frac{m}{2}}}{|\operatorname{Im} z|}, \quad z \notin \mathbb{R} .
$$

In Section 7 we shall give a number of examples of non-self-adjoint operators which have no basis of (generalized) eigenvectors. This will immediately follow upon demonstrating that the norms of their eigenprojectors diverge or that the pseudospectrum of $H$ does not obey the restriction (21). The resolution of the identity, which plays a central role in quantum mechanics, is simply not available.

- Completeness. There exist weaker notions of basis in the literature (e.g. Abel-Lidskii basis), but they are not nearly as useful in applications. The weakest is to merely require the completeness of $\left\{\psi_{k}\right\}_{k=1}^{\infty}$, i.e. that the span of $\left\{\psi_{k}\right\}_{k=1}^{\infty}$ is dense in $\mathcal{H}$, or equivalently $\left(\operatorname{span}\left\{\psi_{k}\right\}_{k=1}^{\infty}\right)^{\perp}=\{0\}$. We have a converse of Proposition 5 for a minimal complete set $\left\{\psi_{k}\right\}_{k=1}^{\infty}$, which implies that the projections $P_{k}$ in (19) may be defined: if the sums of projectors $\sum_{k=1}^{N} P_{k}$ are uniformly bounded, then $\left\{\psi_{k}\right\}_{k=1}^{\infty}$ is a basis, $c f$. [26, Lem. 3.3.3].

\section{Examples}

In this last section we present a number of examples exhibiting remarkable pseudospectral behaviour due to non-Hermiticity and use them to demonstrate that the concept of pseudospectrum is a more relevant consideration for the description of the operators, specifically in the context of quantum mechanics.

We restrict ourselves mainly to the concrete situation of one-dimensional differential operators familiar from "non-Hermitian quantum mechanics". However, we emphasize that there exist versions of Theorem 1 for partial differential (even pseudodifferential) operators too (see [81, 29]) and it is straightforward to 
construct similar examples with non-trivial pseudospectra in higher dimensions. Non-Hermitian spectral effects for $\mathcal{P} \mathcal{T}$-symmetric waveguides were previously observed in [19, 49, 20].

To avoid complicated notation, we study an operator $H$ which changes in each subsection. Where there is a parameter dependence, we may write a subscript as in $H_{h}$. The notation $H_{\text {sa }}$ will denote a self-adjoint operator, related to $H$ usually via a formal (unbounded) conjugation. The symbol $C$ (occasionally with a subscript) will denote a generic constant which may change from line to line.

\subsection{The imaginary Airy operator}

The non-self-adjoint operator

$$
H:=-\frac{\mathrm{d}^{2}}{\mathrm{~d} x^{2}}+i x \quad \text { on } \quad L^{2}(\mathbb{R})
$$

arises in the Ginzburg-Landau model of superconductivity [10, 12, 11, and also in the study of resonances of quantum Hamiltonians with electric field via the method of complex scaling [40]. It is well defined as a closed operator when considered on its maximal domain

$$
\operatorname{Dom}(H):=\left\{\psi \in L^{2}(\mathbb{R}) \mid-\psi^{\prime \prime}+i x \psi \in L^{2}(\mathbb{R})\right\} \text {. }
$$

Indeed, such a definition coincides with the closure of (22) initially defined on smooth functions of compact support, $c f$. 33, Cor. VII.2.7]. More importantly, $H$ is $m$-accretive, i.e., the numerical range $\operatorname{Num}(H)$ is contained in the closed right complex half-plane and the resolvent bound $\left\|(H-z)^{-1}\right\| \leq|\operatorname{Re} z|^{-1}$ holds for all $z$ with $\operatorname{Re} z<0$. The adjoint $H^{*}$ of $H$ is simply obtained by replacing $i$ with $-i$ in (22) and (23). Furthermore, $H$ is $\mathcal{P} \mathcal{T}$-symmetric and $\mathcal{T}$-self-adjoint.

- Spectrum. Integrating by parts, we easily check that

$$
\begin{aligned}
\left\|\psi^{\prime}\right\|^{2} & =\left\langle\psi^{\prime}, \psi^{\prime}\right\rangle=\left\langle\psi,-\psi^{\prime \prime}\right\rangle \leq\|\psi\|\left\|\psi^{\prime \prime}\right\| \leq \delta\left\|\psi^{\prime \prime}\right\|^{2}+\delta^{-1}\|\psi\|^{2}, \\
\|H \psi\|^{2} & =\left\|\psi^{\prime \prime}\right\|^{2}+\|x \psi\|^{2}+2 \operatorname{Re}\left\langle i x \psi,-\psi^{\prime \prime}\right\rangle=\left\|\psi^{\prime \prime}\right\|^{2}+\|x \psi\|^{2}+2 \operatorname{Re}\left\langle i \psi, \psi^{\prime}\right\rangle \\
& \geq\left\|\psi^{\prime \prime}\right\|^{2}+\|x \psi\|^{2}-2\|\psi\|\left\|\psi^{\prime}\right\| \geq\left\|\psi^{\prime \prime}\right\|^{2}+\|x \psi\|^{2}-\delta\left\|\psi^{\prime}\right\|^{2}-\delta^{-1}\|\psi\|^{2},
\end{aligned}
$$

for every $\psi \in C_{0}^{\infty}(\mathbb{R})$ and $\delta>0$. Combining these inequalities for $\delta>0$ sufficiently small and using the density of $C_{0}^{\infty}(\mathbb{R})$ in $\operatorname{Dom}(H)$, we arrive at the non-trivial fact that

$$
\operatorname{Dom}(H)=\left\{\psi \in W^{2,2}(\mathbb{R}) \mid x \psi \in L^{2}(\mathbb{R})\right\} .
$$

Here $W^{2,2}(\mathbb{R})$ denotes the usual Sobolev space of functions in $L^{2}(\mathbb{R})$ whose weak first and second derivatives belong to $L^{2}(\mathbb{R}), c f$. 3 . Now it is clear that $\operatorname{Dom}(H)$ is compactly embedded in $L^{2}(\mathbb{R})$ and $H$ is an operator with compact resolvent, cf. [62, Thm. XIII.65]. It follows that the spectrum of $H$ may consist of isolated eigenvalues only. However, the eigenvalue equation $H \psi=\lambda \psi$ implies that for any $c \in \mathbb{R}$ we also have $H \psi_{c}=\lambda_{c} \psi_{c}$ with $\psi_{c}(x):=\psi(x+c)$ and $\lambda_{c}:=\lambda-i c$. Consequently, the spectrum of $H$ is empty,

$$
\sigma(H)=\varnothing .
$$


This is a peculiar property, possible for non-self-adjoint operators only. We deduce that $H$ is not similar to a self-adjoint operator, via a bounded and boundedly invertible similarity transform.

- Pseudospectrum. While $H$ has no spectrum, the pseudospectrum of $H$ is far from trivial. A priori, we only know that the pseudospectrum is symmetric with respect to the real axis, i.e. (11) holds, which follows from the $\mathcal{P} \mathcal{P}$-symmetry of $H$. In order to apply Theorem 1 , we have to convert $H$ into a semiclassical operator. This can be achieved by introducing the unitary transform $\mathcal{U}$ on $L^{2}(\mathbb{R})$ defined by

$$
(\mathcal{U} \psi)(x):=\tau^{1 / 2} \psi(\tau x),
$$

where $\tau \in \mathbb{R}$ is positive (and typically large in the sequel). Then

$$
\mathcal{U H \mathcal { U } ^ { - 1 }}=\tau H_{h} \quad \text { with } \quad H_{h}:=-h^{2} \frac{\mathrm{d}^{2}}{\mathrm{~d} x^{2}}+i x \quad \text { and } \quad h:=\tau^{-3 / 2} .
$$

For the symbol $f=\xi^{2}+i x$ associated with $H_{h}$ we have $\{f, \bar{f}\}=-4 i \xi$. Hence, the interior of the semiclassical pseudospectrum is $\Lambda=\{z \in \mathbb{C} \mid \operatorname{Re} z>0\}$, using definition (15).

The same translation argument which shows that the spectrum is empty shows that

$$
\left\|(H-z)^{-1}\right\|=\left\|(H-\operatorname{Re} z)^{-1}\right\| .
$$

Note that $1 \in \Lambda$. Applying the unitary relation and Theorem 1, there exists some $C>1$ where, for $h$ sufficiently small (that is, $\tau>C_{1}$ for some $C_{1}>0$ sufficiently large),

$$
\left\|(H-\tau)^{-1}\right\|=\tau^{-1}\left\|\left(H_{h}-1\right)^{-1}\right\|>h^{2 / 3} C^{1 / h} .
$$

We then have that $\tau \in \sigma_{\varepsilon}(H)$ whenever $\tau^{-1} C^{\tau^{3 / 2}}>\varepsilon^{-1}$. We may simplify the inequality by taking logarithms: it reads

$$
\tau^{3 / 2}-\frac{\log \tau}{\log C}>\frac{1}{\log C} \log \frac{1}{\varepsilon} .
$$

Since $\log \tau$ is negligible compared with $\tau^{3 / 2}$ for $\tau>0$ large, a sufficient condition to guarantee that $\tau \in \sigma_{\varepsilon}(H)$ is given by

$$
\tau>C_{2}\left(\log \frac{1}{\varepsilon}\right)^{2 / 3}
$$

with some $C_{2}>0$; this gives the correct order of growth as $\varepsilon \rightarrow 0$.

Since the resolvent norm only depends on the real part of the spectral parameter $z$, we arrive at the conclusion that there exist $C_{1}, C_{2}>0$ such that, for all $\varepsilon>0$,

$$
\sigma_{\varepsilon}(H) \supseteq\left\{z \mid \operatorname{Re} z \geq C_{1} \& \operatorname{Re} z \geq C_{2}\left(\log \frac{1}{\varepsilon}\right)^{2 / 3}\right\} .
$$

In particular, for any $\varepsilon$ there are complex points with positive real part and magnitude only logarithmically large in $1 / \varepsilon$ that lie in the pseudospectrum $\sigma_{\varepsilon}(H)$.

A quite precise study of the resolvent norm of $H$ as $\operatorname{Re} z \rightarrow \infty$ can be found in [18, Cor. 1.4]. 
- Time evolution. Since $H$ is $m$-accretive, it is a generator of a one-parameter contraction semigroup, $e^{-t H}$, on $L^{2}(\mathbb{R})$. Here $t$ can be interpreted as time, viewing $\psi(t, x)=e^{-t H} \psi(0, x)$ as arising as a solution of the parabolic equation $\partial_{t} \psi+H \psi=0$. Using the Fourier transform, it is possible to show (cf. [26. Ex. 9.1.7]) that

$$
\left\|e^{-t H}\right\|=e^{-t^{3} / 12} .
$$

Note that the time decay rate is not determined by the (nonexistent) spectrum of $H$. In fact, the superexponential decay rate implies by itself that the spectrum of $H$ must be empty. We refer to [12 for pseudospectral estimates on the decay of the semigroup in analogous higher-dimensional models.

\subsection{The imaginary cubic oscillator}

The non-self-adjoint (but $\mathcal{T}$-self-adjoint) operator

$$
H:=-\frac{\mathrm{d}^{2}}{\mathrm{~d} x^{2}}+i x^{3} \quad \text { on } \quad L^{2}(\mathbb{R})
$$

is considered the fons et origo of $\mathcal{P} \mathcal{T}_{\text {-symmetric quantum mechanics [15, 16, }}$, but it was also considered previously in the context of statistical physics and quantum field theory 34. The existence of a metric operator and other spectral and pseudospectral properties of $H$ have been recently analysed in [71. Let us recall the basic results here, referring to the last article for more details and references.

- Spectrum. The operator $H$ is again $m$-accretive when considered on its maximal domain (i.e. (23) with $x$ replaced by $x^{3}$ ) and its resolvent is compact. Contrary to the imaginary Airy operator, the spectrum is not empty; it is composed of an infinite sequence of discrete real eigenvalues, $c f$. 69, 32, 35. As a new result, it is proved in 71 that the eigenfunctions form a complete set in $L^{2}(\mathbb{R})$.

- Pseudospectrum. Employing the unitary transform (25), we introduce a semiclassical analogue of $H$,

$$
\mathcal{u} H \mathcal{U}^{-1}=\tau^{3} H_{h} \quad \text { with } \quad H_{h}:=-h^{2} \frac{\mathrm{d}^{2}}{\mathrm{~d} x^{2}}+i x^{3} \quad \text { and } \quad h:=\tau^{-5 / 2} .
$$

For the symbol $f$ associated with $H_{h}$ we now have $\{f, \bar{f}\}=-12 i \xi x^{2}$, so that the interior of the semiclassical pseudospectrum is $\Lambda=\{z \in \mathbb{C} \mid \operatorname{Re} z>0 \& \operatorname{Im} z \neq$ $0\}$.

The translation argument used for the imaginary Airy operator is unavailable for the imaginary cubic oscillator, but we nonetheless have by Theorem 1 for any $z \in \Lambda$, there exists $C>0$ sufficiently large and $h_{0}$ sufficiently small that, for all $0<h \leq h_{0}$,

$$
\left\|\left(H-\tau^{3} z\right)^{-1}\right\|=\tau^{-3}\left\|\left(H_{h}-z\right)^{-1}\right\|>h^{6 / 5} C^{1 / h} .
$$

One may check that the exponential growth in Theorem 1 may be made uniform on compact subsets $K \subset \Lambda$, as explained at the end of Appendix $\mathrm{A}$. What is 
more, one may extend the reasoning to include real $z>0$, despite the fact that formally $z \notin \Lambda$ : one only needs to verify (87) by hand, which is straightforward.

Since we are using a scaling argument, we may reduce to fixing $\delta>0$ and letting

$$
K=\{z \in \mathbb{C}|| z|=1 \&| \arg z \mid<\pi / 2-\delta\} .
$$

We therefore have that, for some positive constant $C$ depending on $\delta$, the pseudospectrum $\sigma_{\varepsilon}(H)$ contains $\tau^{3} K$ so long as $\tau>0$ is large enough to verify $\tau^{-3} C^{\tau^{5 / 2}}>\varepsilon^{-1}$. We may then identify $\tau^{3}$ with the absolute value of the spectral parameter, and so $h=\tau^{-5 / 2}=|z|^{-5 / 6}$. Taking logarithms and discarding the negligible term involving logarithms, as before, allows us to conclude that, for any $\delta>0$, there exist $C_{1}, C_{2}>0$ such that, for all $\varepsilon>0$,

$$
\sigma_{\varepsilon}(H) \supseteq\left\{z \in \mathbb{C}|| z\left|\geq C_{1} \&\right| \arg z\left|<\left(\frac{\pi}{2}-\delta\right) \&\right| z \mid \geq C_{2}\left(\log \frac{1}{\varepsilon}\right)^{6 / 5}\right\} .
$$

Again, for any $\varepsilon$ there are complex points with positive real part, non-zero imaginary part, and large magnitude that lie in the pseudospectrum $\sigma_{\varepsilon}(H)$. A numerical computation of some pseudospectral lines of $H$ is presented in Figure 1. The asymptotic behaviour of the pseudospectral lines is studied in [18, Prop. 4.1]. The result is surprising because it implies the existence of

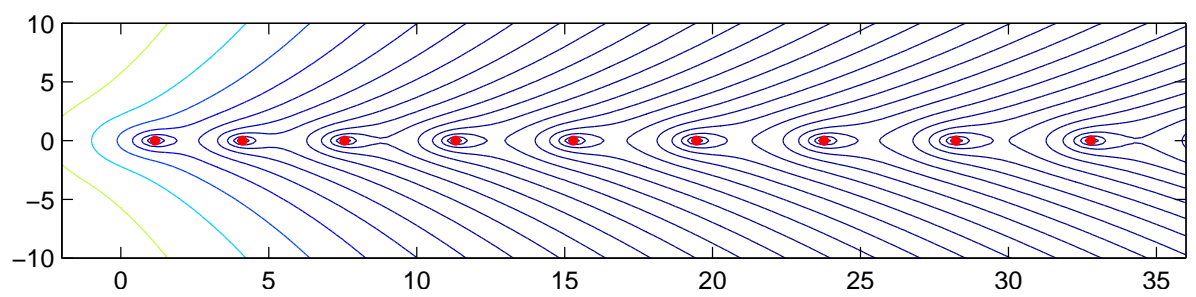

Figure 1: Spectrum (red dots) and pseudospectra (enclosed by the blue contour lines) of the imaginary cubic oscillator.

pseudoeigenvalues very far from the spectrum of $H$. In view of (5), it follows that a very small perturbation $V$ added to $H$ can create (genuine) eigenvalues very far from the spectrum of the unperturbed operator $H$. In this way, the spectrum is highly unstable.

As a consequence of the existence of the highly non-trivial pseudospectrum, we also get that $H$ is not quasi-Hermitian with a bounded and boundedly invertible metric (Proposition 3), it is not similar to a self-adjoint operator via bounded and boundedly invertible transformations (Proposition 2), and the eigenfunctions of $H$ do not form a Riesz basis (Proposition 4). It has been shown recently in [39] that the norms of the spectral projections grow as

$$
\lim _{k \rightarrow \infty} \frac{\log \left\|P_{k}\right\|}{k}=\frac{\pi}{\sqrt{3}}
$$

and therefore the eigenfunctions cannot form a basis, $c f$. Proposition 5 . Alternatively, we can derive the latter using Proposition 6 and (27). 


\subsection{An advection-diffusion operator}

The examples which follow are similar to self-adjoint operators via unbounded transformations. In order to emphasize the danger of formal manipulations when the transformations are allowed to be unbounded, in this subsection we present a very simple non-self-adjoint operator for which heuristic approaches would lead to a number of striking paradoxes. The example is borrowed from [60, 25], although it is natural to expect that it has appeared in many other works. We are indebted to E. B. Davies and M. Marletta 28 for telling us about this example and for proposing the possibility of the non-invariance of point spectra discussed below.

Consider the differential operator

$$
H:=-\frac{\mathrm{d}^{2}}{\mathrm{~d} x^{2}}+\frac{\mathrm{d}}{\mathrm{d} x} \quad \text { on } \quad L^{2}(\mathbb{R}) .
$$

The diffusion term $-\mathrm{d}^{2} / \mathrm{d} x^{2}$ corresponds to the familiar free Hamiltonian in quantum mechanics, which is self-adjoint when defined on $W^{2,2}(\mathbb{R})$. The advection term represents a relatively bounded perturbation with relative bound equal to zero, so that $\operatorname{Dom}(H)=W^{2,2}(\mathbb{R})$, cf. [44, Sec. IV.1.1]. Employing the first line of (24), we find

$$
|\operatorname{Im}(\psi, H \psi)| \leq\|\psi\|\left\|\psi^{\prime}\right\|=\|\psi\| \sqrt{\operatorname{Re}(\psi, H \psi)}
$$

for every $\psi \in \operatorname{Dom}(H)$. It follows that the numerical range $\operatorname{Num}(H)$ is contained in the parabolic domain $\Sigma:=\left\{\left.z \in \mathbb{C}|\operatorname{Re} z \geq 0 \&| \operatorname{Im} z\right|^{2} \leq \operatorname{Re} z\right\}$. In particular, $H$ is not only $m$-accretive but even $m$-sectorial, meaning that, in addition, its numerical range is a subset of a sector in the complex plane. By conjugating with the Fourier transform, it is easy to check that the spectrum of $H$ coincides with the parabola

$$
\sigma(H)=\partial \Sigma=\left\{\left.z \in \mathbb{C}|\operatorname{Re} z \geq 0 \&| \operatorname{Im} z\right|^{2}=\operatorname{Re} z\right\},
$$

see Figure 2 .

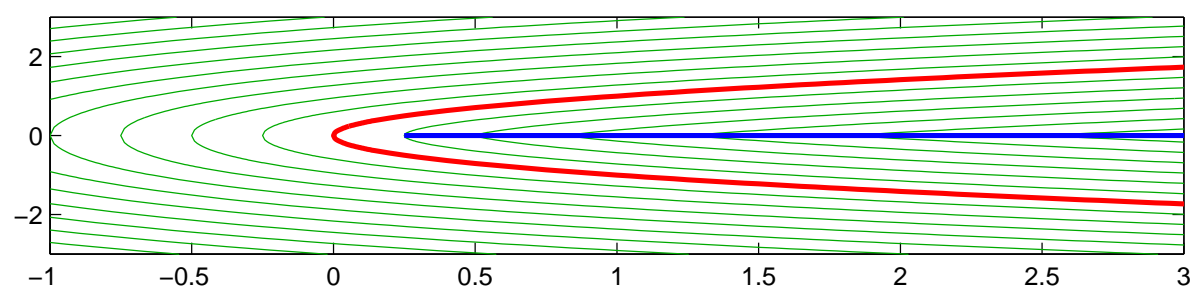

Figure 2: Spectrum of the advection-diffusion operator (red parabola) compared with the spectrum of the shifted free Hamiltonian (blue line) to which the former is formally similar and pseudospectral contours (green curves parallel to the parabola).

- Non-invariance of the continuous spectrum. Completing the square, we may write

$$
H=-\left(\frac{\mathrm{d}}{\mathrm{d} x}-\frac{1}{2}\right)^{2}+\frac{1}{4} \text {. }
$$


This suggests that the similarity transformation $\Omega:=e^{-x / 2}$ formally maps $H$ to a shifted free Hamiltonian

$$
H_{\mathrm{sa}}:=-\frac{\mathrm{d}^{2}}{\mathrm{~d} x^{2}}+\frac{1}{4}
$$

which is self-adjoint on $\operatorname{Dom}\left(H_{\mathrm{sa}}\right):=W^{2,2}(\mathbb{R})$. The word "formally" is absolutely essential here, since neither $\Omega$ nor $\Omega^{-1}$ is bounded and (2) cannot hold as an operator identity. Nevertheless, one can check that (2) holds on smooth functions with compact support, which form a dense subset of both $\operatorname{Dom}(H)$ and $\operatorname{Dom}\left(H_{\mathrm{sa}}\right)$. Now we arrive at a surprising paradox because

$$
\sigma\left(H_{\mathrm{sa}}\right)=\left[\frac{1}{4}, \infty\right)
$$

substantially differs from the complex parabolic spectrum of $H$, see Figure 2. This is caused by the fact that the continuous spectrum is in general not preserved by unbounded similarity transformations.

- Non-invariance of the point spectrum. The situation is in fact even worse, since it may also happen that even the eigenvalues are not preserved by the similarity transformation $\Omega$. Let us perturb the self-adjoint Hamiltonian $H_{0}$ by a smooth non-trivial potential $V \leq 0$ which has a compact support in $\mathbb{R}$. Then it is well known (see, e.g., 65, Cor. 4.5.2]) that the self-adjoint operator $H_{\mathrm{sa}}+V$ possesses at least one eigenvalue $\lambda<1 / 4$. The corresponding eigenfunction $\psi$ has asymptotics $\exp (-\sqrt{1 / 4-\lambda}|x|)$ as $x \rightarrow \pm \infty$. It is also known that $\lambda$ is positive provided that the supremum norm of $V$ is sufficiently small, $c f$. 72 . However, the corresponding "eigenfunction" $\Omega^{-1} \psi$ of $H+V$ is not admissible because it is not square-integrable on $\mathbb{R}$.

- Pseudospectrum. Even if $H$ is not self-adjoint (nor $\mathcal{T}$-self-adjoint or $\mathcal{P} \mathcal{T}$ symmetric), it is normal (and in fact real). Hence the pseudospectra are trivial; see Figure 2. The situation would be very different if we considered (30) on a finite interval $(0, L)$, subject to Dirichlet boundary conditions. Let us denote this operator by $H^{(L)}$. Then the similarity transformation $\Omega=e^{-x / 2}$ is bounded and boundedly invertible and (2) with the self-adjoint operator $H_{\mathrm{sa}}^{(L)}$ that acts as (31) on $(0, L)$, subject to Dirichlet boundary conditions, is well defined. We indeed have

$$
\sigma\left(H^{(L)}\right)=\sigma\left(H_{\mathrm{sa}}^{(L)}\right)=\left\{\left(\frac{\pi k}{L}\right)^{2}+\frac{1}{4} \mid k=1,2, \ldots\right\} .
$$

However, the pseudospectra of $H^{(L)}$ substantially differ from those of $H_{\mathrm{sa}}^{(L)}$; the former approach the parabola $\partial \Sigma$ in the limit as $L \rightarrow \infty$, thus reflecting better the wild spectral instability of the limit; see Figure 2. We refer to [60] for more details.

\subsection{The rotated harmonic oscillator}

The quantum Hamiltonian of the harmonic oscillator

$$
H_{\mathrm{sa}}:=-\frac{\mathrm{d}^{2}}{\mathrm{~d} x^{2}}+x^{2} \quad \text { on } \quad L^{2}(\mathbb{R})
$$


is self-adjoint on its maximal domain. The operator $H_{\mathrm{sa}}$ has compact resolvent and its eigenvalues are well-known:

$$
\sigma\left(H_{\mathrm{sa}}\right)=\{2 k+1 \mid k=0,1, \ldots\} .
$$

Of course, the pseudospectrum of $H_{\mathrm{sa}}$ is trivial; see Figure 4

- Creation and annihilation operators. Recall also the factorization $H_{\mathrm{sa}}=$ $a^{*} a+1$, where $a^{*}$ and $a$ are the creation and annihilation operators defined by

$$
a^{*}:=-\frac{\mathrm{d}}{\mathrm{d} x}+x, \quad a:=\frac{\mathrm{d}}{\mathrm{d} x}+x,
$$

with $\operatorname{Dom}(a)=\operatorname{Dom}\left(a^{*}\right):=W^{1,2}(\mathbb{R}) \cap L^{2}\left(\mathbb{R}, x^{2} \mathrm{~d} x\right)$. As the notation suggests, $a^{*}$ and $a$ are mutually adjoint. The operator domain $\operatorname{Dom}(a)$ in fact coincides with the form domain of $H_{\mathrm{sa}}$, for which $C_{0}^{\infty}(\mathbb{R})$ is a core. We incidentally remark that $a^{*}$ and $a$ represent interesting examples of highly non-self-adjoint operators for which the resolvent operator is not defined at any point of the complex plane:

$$
\sigma\left(a^{*}\right)=\sigma(a)=\mathbb{C} .
$$

Indeed, $\psi(x):=\exp \left(\lambda x-x^{2} / 2\right)$ is an eigenfunction of $a$ for every $\lambda \in \mathbb{C}$. On the other hand, the point spectrum of $a^{*}$ is empty, but every complex point belongs to the residual spectrum of $a^{*}$. The latter follows by the general fact that the orthogonal complement of the range of a densely defined closed operator in a Hilbert space is equal to the kernel of its adjoint.

- Complex dilation. The rotated harmonic oscillator $H$ is formally obtained by the similarity transformation (2), where $\Omega^{-1}$ is the complex dilation operator defined by $\left(\Omega^{-1} \psi\right)(x):=e^{i \theta / 4} \psi\left(e^{i \theta / 2} x\right)$. If $\theta$ were purely imaginary, $\Omega^{-1}$ would be unitary, but we assume $\theta \in \mathbb{R}$ when $\Omega^{-1}$ is in fact unbounded. The formal computation yields

$$
H=-e^{-i \theta} \frac{\mathrm{d}^{2}}{\mathrm{~d} x^{2}}+e^{i \theta} x^{2}=e^{-i \theta}\left(-\frac{\mathrm{d}^{2}}{\mathrm{~d} x^{2}}+e^{2 i \theta} x^{2}\right) \quad \text { on } \quad L^{2}(\mathbb{R}),
$$

which we take as a definition and restrict to $|\theta|<\pi / 2$. The operator $H$ is sometimes referred to as Davies' oscillator due to his important investigation 22] (see also [26, Sec. 14.5] for a summary and other references). The presence of the prefactor $e^{-i \theta}$ in our definition is inessential, but it is useful for symmetry reasons. In particular, we have

$$
|\operatorname{Im}(\psi, H \psi)| \leq|\tan \theta| \operatorname{Re}(\psi, H \psi)=|\sin \theta|\left(\psi, H_{\mathrm{sa}} \psi\right)
$$

for every $\psi \in C_{0}^{\infty}(\mathbb{R})$. That is, under our restriction on $\theta$, the operator $H$ can be understood as obtained as a relatively form bounded perturbation of $H_{\mathrm{sa}}$, keeping the same form domain $\operatorname{Dom}(a)$. The numerical range $\operatorname{Num}(H)$ lies in the symmetric sector $\{z \in \mathbb{C}|| \operatorname{Im} z|\leq| \tan \theta \mid \operatorname{Re} z \& \operatorname{Re} z \geq 0\}$. Unless $\theta=0$, $H$ is neither self-adjoint nor $\mathcal{P} \mathcal{T}$-symmetric, but it is always $\mathcal{T}$-self-adjoint. 
- Spectrum and pseudospectrum. The resolvent of $H$ is again compact and the spectrum coincides with that of $H_{\mathrm{sa}}$ :

$$
\sigma(H)=\sigma\left(H_{\mathrm{sa}}\right) .
$$

In particular, the spectrum is real. However, the pseudospectrum and basis properties of the eigenfunctions are very different from the self-adjoint situation. In the same way as we applied Theorem 1 to the imaginary Airy operator or cubic oscillator, we find

$$
\Lambda=\{z \in \mathbb{C} \backslash\{0\}|| \arg z \mid<\theta\} .
$$

When $\theta=0, \Lambda$ is empty and $H_{\mathrm{sa}}$ is self-adjoint, so that we know that its pseudospectrum is trivial. If $\theta \neq 0$, however, we have exponential growth for the resolvent indicated by Theorem 1 which may be made uniform for a compact subset contained in the interior of the semiclassical pseudospectrum. What is more, from [41, Thm. 1.1, Rem. 1.3], we have upper bounds for the resolvent norm of essentially the same exponential type, though the gap in the constant leaves much to be understood about the precise behaviour.

A scaling argument similar to that used for (28) gives us an idea of size of the $\varepsilon$-pseudospectrum, which includes more or less those $z \in \operatorname{Num}(H)$ for which $|z|>C \log (1 / \varepsilon)$, which grows very slowly as $\varepsilon \rightarrow 0$.

Specifically, for any $\delta>0$, we have from Theorem 1 that there exist $C_{1}, C_{2}>$ 0 such that, for all $\varepsilon>0$,

$$
\sigma_{\varepsilon}(H) \supseteq\left\{z|| z\left|>C_{1} \&\right| \arg z|\leq \theta-\delta \&| z \mid>C_{2} \log \frac{1}{\varepsilon}\right\} .
$$

The numerical range inequality (4) gives that

$$
\sigma_{\varepsilon}(H) \subseteq\{z \mid \operatorname{dist}(z, \operatorname{Num}(H))<\varepsilon\} .
$$

What is more, rescaling the upper bound [41, Eq. (1-8)] gives that there exists $C_{3}>0$ for which

$$
\left\|(H-z)^{-1}\right\| \leq \frac{C_{3} e^{C_{3}|z|}}{\operatorname{dist}(z, \sigma(H))} .
$$

We therefore see that $z$ cannot be in the pseudospectrum unless it is logarithmically large in $1 / \varepsilon$ or close to the spectrum:

$$
\sigma_{\varepsilon}(H) \subseteq\left\{z|| z \mid>\frac{1}{C_{3}}\left(\log \frac{1}{\varepsilon}-\log C_{3}\right)\right\} \cup\left\{z \mid \operatorname{dist}(z, \sigma(H))<C_{3} \varepsilon e^{C_{3}|z|}\right\} .
$$

In Figure 3, we have a diagram illustrating the sorts of regions containing and contained by the pseudospectrum. We emphasize that the constants involved were chosen by hand and do not reflect a precise application of the relevant theorems. As in the previous examples, for any $\varepsilon>0$ there are complex points with positive real part, non-zero imaginary part, and large magnitude that lie in the pseudospectrum $\sigma_{\varepsilon}(H)$; see Figure 4 The asymptotic behaviour of the pseudospectrum has been also studied in [56, 18]. 


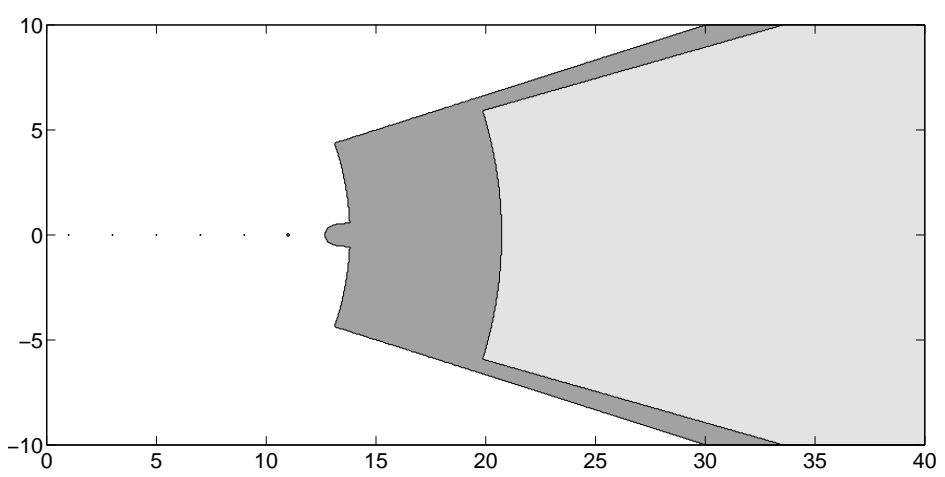

Figure 3: Diagram of region (dark gray) which contains $\sigma_{\varepsilon}(H)$ and region with pseudomodes (light gray) which is definitely contained in $\sigma_{\varepsilon}(H)$.

- Wild basis properties. $H_{0}$ is self-adjoint and we know a priori that its eigenfunctions (after normalization) form a complete orthonormal family in $L^{2}(\mathbb{R})$. If $\theta \neq 0$, it is still true that the eigenfunctions of $H$ and $H^{*}$ (after a suitable normalization) form a biorthonormal sequence. However, the eigenfunctions do not form a Riesz basis or even a basis, as can be deduced from the non-trivial pseudospectrum ( $c f$. Propositions 4 and 6).

Furthermore, it was shown in 27] that the norms of the spectral projections of $H$ associated with eigenvalues $2 k+1$ grow exponentially as $k \rightarrow \infty$, and the exact exponential rate of growth was identified. This exponential growth rate was sharpened in [38, Thm. 1.2, Rem. 1.3] to include an asymptotic expansion for the remainder and a generalization to operators including $-\mathrm{d}^{2} / \mathrm{d} x^{2}+e^{2 i \theta}|x|^{2 m}$ for $m \in \mathbb{N}$ and natural restrictions on $\theta$.

In the case of the rotated harmonic oscillator, one has from [79, Cor. 1.7, Ex. 3.6] an exact integral formula leading to a similar asymptotic expansion and a simplification of the formula for the exponential growth rate to

$$
\lim _{k \rightarrow+\infty} \frac{\log \left\|P_{k}\right\|}{k}=\frac{1}{2} \log \left(\frac{1+|\sin \theta|}{1-|\sin \theta|}\right) .
$$

The new and very short proof of this formula using special functions is given in Appendix B

Summing up, despite the reality of the spectrum of $H$ and the existence of a formal similarity transformation given by a complex dilation, we see that $H$ exhibits very different properties from those enjoyed by self-adjoint operators.

\subsection{The shifted harmonic oscillator}

The shift operator $T_{t}$ is, for $t \in \mathbb{R}$, well defined as a bounded operator on $L^{2}(\mathbb{R})$ by the formula $\left(T_{t} \psi\right)(x):=\psi(x+t)$. It is in fact a unitary group $T_{t}=e^{t \mathrm{~d} / \mathrm{d} x}$ with the self-adjoint generator $-i \mathrm{~d} / \mathrm{d} x$ defined on $W^{1,2}(\mathbb{R})$ being the familiar momentum operator in quantum mechanics. Let $H_{\mathrm{sa}}$ be again the self-adjoint harmonic oscillator (32) and perform the formal conjugation (2) with the unbounded operator $\Omega:=T_{-i}$. Then we arrive at the non-self-adjoint operator

$$
H=-\frac{\mathrm{d}^{2}}{\mathrm{~d} x^{2}}+(x+i)^{2} \quad \text { on } \quad L^{2}(\mathbb{R}),
$$


which we again take as a definition. The extra term $2 i x-1$ clearly represents a relatively bounded perturbation of $H_{\mathrm{sa}}$ with the relative bound equal to zero, so that $\operatorname{Dom}(H)=\operatorname{Dom}\left(H_{\mathrm{sa}}\right)$. It is also relatively form bounded with relative bound equal to zero, so that $H$ is $m$-sectorial on $\operatorname{Dom}(a)$. The operator $H$ is $\mathcal{P}$-symmetric and $\mathcal{T}$-self-adjoint.

- Spectrum and pseudospectrum. The above remarks on the smallness of the perturbations imply that the resolvent of $H$ is compact. Notice that conjugation with the Fourier transform casts $H$ into a unitarily equivalent operator

$$
\hat{H}:=\left(-i \frac{\mathrm{d}}{\mathrm{d} x}-i\right)^{2}+x^{2},
$$

which is related to (32) via the unbounded similarity transform $\hat{\Omega} \psi(x):=$ $e^{x} \psi(x)$. Using that the eigenfunctions of the harmonic oscillator (32) are known to decay superexponentially, it can be showed that

$$
\sigma(H)=\sigma\left(H_{\mathrm{sa}}\right)
$$

analogously to (36).

Numerical computations show that the pseudospectrum of $H$ is non-trivial; see Figure 4. Because of the different scaling properties of $x^{2}$ and $x$, Theorem 1 is not directly applicable. Nonetheless, we show, by adapting the construction of pseudomodes, that there are indeed large complex pseudoeigenvalues in parabolic regions of the complex plane.

Theorem 7. Let $H$ be the operator from (38). Fix $\varepsilon>0$. Then there exists $C>0$ sufficiently large such that for all $z \in \mathbb{C}$ for which $\operatorname{Re} z>C$ and

$$
|\operatorname{Im} z| \leq 2(1-\varepsilon) \sqrt{\operatorname{Re} z}
$$

we have the resolvent lower bound

$$
\left\|(H-z)^{-1}\right\| \geq \frac{1}{C} e^{\sqrt{\operatorname{Re} z} / C} .
$$

We postpone the proof to Appendix $\mathrm{A}$

- Wild basis properties. The pseudospectral criteria of Propositions 3, 4 and 6 show that the eigenfunctions of $H$ cannot form a Riesz basis or even a basis. Moreover, explicit formulas for the eigenfunctions can be used to prove their completeness in $L^{2}(\mathbb{R})$ and to find the rate of the norms of spectral projections, namely

$$
\lim _{k \rightarrow+\infty} \frac{\log \left\|P_{k}\right\|}{\sqrt{k}}=2^{3 / 2},
$$

see [51, Sec. 2] for the detailed proof.

\subsection{The decaying and singular potential perturbations of harmonic oscillator}

Examples in previous sections show that the perturbations of harmonic oscillator may have very non-trivial pseudospectra and wild basis properties, although 


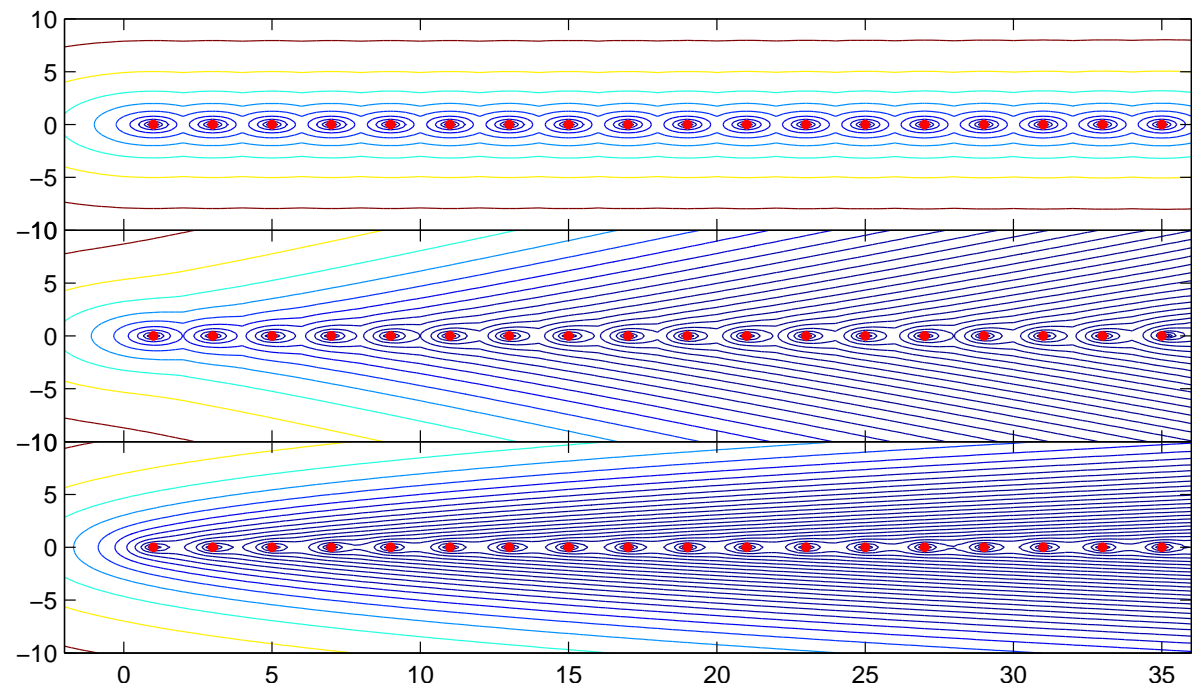

Figure 4: From top to bottom: Pseudospectra of the self-adjoint (32), rotated (35) with $\theta=\pi / 4$ and shifted (38) harmonic oscillators which are formally conjugate to each other. Although the spectra (red dots) coincide in all these examples, the pseudospectra exhibit striking differences.

the spectrum is preserved. In order not to leave an impression that this is typical for any perturbation of the harmonic oscillator, we mention that the system of all generalized eigenfunctions of perturbations $H=H_{\mathrm{sa}}+V$ of $H_{\mathrm{sa}}$ in (32) contains a Riesz basis when the perturbation $V$ is multiplication by a function satisfying, for instance, $V \in L^{p}(\mathbb{R}), 1 \leq p<\infty$, or $V$ is a finite linear combination of $\delta$-potentials with complex couplings; $c f$. [4, 5, 50, for details and additional examples. It is also showed in these works that the eigenvalues of the perturbed operator $H$, excluding possibly a finite number, remain simple. Therefore, if the perturbation satisfies some symmetries, e.g. it is $\mathcal{P} \mathcal{T}$-symmetric, we can conclude that the eigenvalues of $H$ are real, again up to a finite number. Moreover, if we insert a sufficiently small coupling constant $\varepsilon$ in front of $V$, we obtain that all eigenvalues are simple and real. When the coupling constant is increased, low lying eigenvalues may collide, create a Jordan block, and then become complex. This behaviour is illustrated in Figure 5 for an example of $H$ where $V$ is $\mathcal{P} \mathcal{T}$-symmetric and has the form (40).

- Pseudospectrum. Let us recall that the facts that the eigensystem contains a Riesz basis and the spectral properties described above imply that $H$ is similar to a diagonal operator up to a possible finite number of finite dimensional Jordan blocks corresponding to the low lying eigenvalues. This in turn means that the pseudospectrum of $H$ is trivial if there are no Jordan blocks. If $\lambda_{0}$ is a degenerate eigenvalue for which the algebraic multiplicity is strictly larger than the geometric one, the Jordan block result in a more singular behaviour of the resolvent, i.e.

$$
\left\|(H-z)^{-1}\right\| \sim\left|\lambda_{0}-z\right|^{-n}, \quad n>1
$$




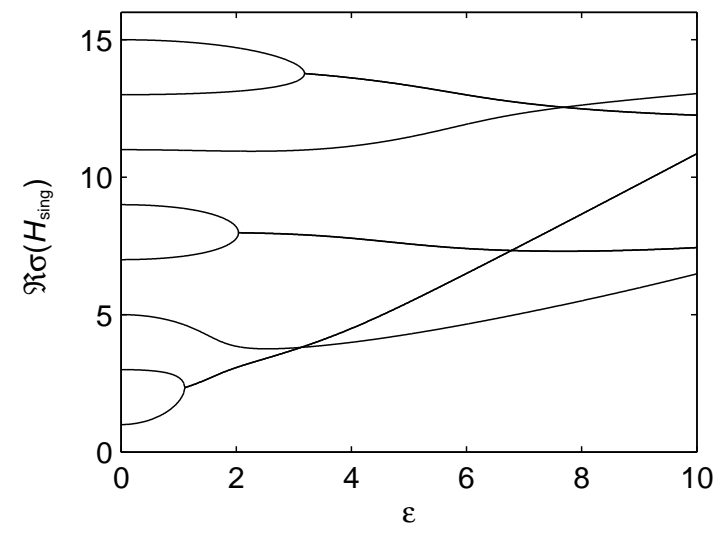

Figure 5: Real parts of eigenvalues of $H$ with (40) as a function of $\varepsilon$.

around $\lambda_{0}$. This is visible in the plot of the pseudospectrum, since the peak around the degenerate eigenvalue $\lambda_{0}$ is wider, i.e. the level lines are further from $\lambda_{0}$, and we can call the pseudospectrum "almost trivial". We illustrate such a behaviour with the example

$$
V(x):=i \varepsilon\left(\frac{1}{\sqrt{|x+1|}}-\frac{1}{\sqrt{|x-1|}}\right),
$$

see Figure 6
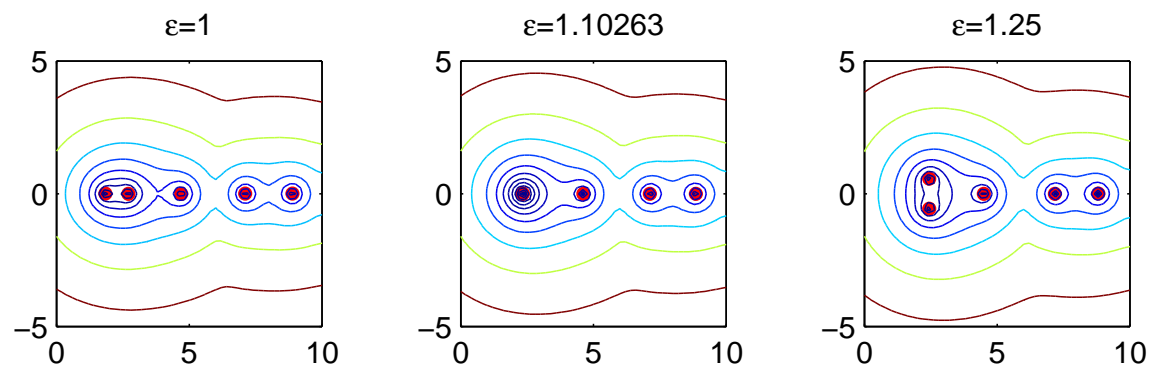

Figure 6: Pseudospectra around low lying eigenvalues of (40) for three increasing values of $\varepsilon$. From left to right: The simple eigenvalues (red dots) collide and create a Jordan block, then become again simple, but complex. The Jordan block structure is indicated by the wider peak around the degenerate eigenvalue.

Similar features appear in for instance a model on a finite interval with $\mathcal{P} \mathcal{T}$ symmetric Robin boundary conditions, $c f$. 47, 46, 48, 45, or for a $\mathcal{P} \mathcal{T}$-symmetric square-well, cf. [80, 70]. 


\subsection{The gauged oscillator (Swanson's model)}

We also study the operator

$$
H:=\omega a^{*} a+\alpha a^{2}+\beta\left(a^{*}\right)^{2}+\omega \text { in } L^{2}(\mathbb{R}),
$$

introduced by Ahmed in 6] and later studied by Swanson in 75. Here the creation and annihilation operators are defined in (34) (the reader is warned that a different convention is used in [75) and $\omega, \alpha, \beta$ are real parameters. It is assumed in 75 , only that

$$
\omega^{2}-4 \alpha \beta \geq 0
$$

but we shall see that this condition is by far insufficient to make the results rigorous.

- Rigorous definition of the operator. First of all, we always assume $\omega \neq 0$ in order to define $H$ as a perturbation of the harmonic oscillator $H_{\text {sa }}$ defined in (32); without loss of generality, let us take $\omega>0$. Second, we need to impose a condition on the smallness of $\alpha$ and $\beta$ in order to ensure that the extra unbounded terms $r:=\alpha a^{2}+\beta\left(a^{*}\right)^{2}$ added in (41) to $\omega a^{*} a=\omega H_{\mathrm{sa}}-\omega$ do not completely change the character of the operator $H_{\mathrm{sa}}$; see Section 7.8 for a discussion of the relevant condition in a more general setting.

Expressing annihilation and creation operators in terms of $x$ and $\mathrm{d} / \mathrm{d} x$, we obtain an equivalent form

$$
H=-(\omega+\alpha+\beta) \frac{\mathrm{d}^{2}}{\mathrm{~d} x^{2}}+(\omega-\alpha-\beta) x^{2}+(\alpha-\beta)\left(\frac{\mathrm{d}}{\mathrm{d} x} x+x \frac{\mathrm{d}}{\mathrm{d} x}\right) \quad \text { on } \quad L^{2}(\mathbb{R}) .
$$

We introduce $H$ as an $m$-sectorial operator with compact resolvent under the condition

$$
\omega-|\alpha+\beta|>0 .
$$

Notice that this condition is stronger than (42) and it ensures that the real part of $H$ indeed resembles the usual harmonic oscillator, because the constants in front of $-\mathrm{d}^{2} / \mathrm{d} x^{2}$ and $x^{2}$ are both positive.

We define a form

$$
\begin{gathered}
t[\psi]:=(\omega+\alpha+\beta)\left\|\psi^{\prime}\right\|^{2}+(\omega-\alpha-\beta)\|x \psi\|^{2} \\
+(\alpha-\beta)\left(\left\langle\psi^{\prime}, x \psi\right\rangle+\left\langle x \psi, \psi^{\prime}\right\rangle\right), \\
\operatorname{Dom}(t):=\operatorname{Dom}(a)=\left\{\psi \in W^{1,2}(\mathbb{R}) \mid x \psi \in L^{2}(\mathbb{R})\right\} .
\end{gathered}
$$

Since the imaginary part of $t$ satisfies

$$
\begin{aligned}
|\operatorname{Im} t[\psi]| & =\left|(\alpha-\beta)\left(\left\langle\psi^{\prime}, x \psi\right\rangle+\left\langle x \psi, \psi^{\prime}\right\rangle\right)\right| \\
& \leq|\alpha-\beta|\left(\left\|\psi^{\prime}\right\|^{2}+\|x \psi\|^{2}\right) \\
& \leq \frac{|\alpha-\beta|}{\omega-|\alpha+\beta|}\left((\omega+\alpha+\beta)\left\|\psi^{\prime}\right\|^{2}+(\omega-\alpha-\beta)\|x \psi\|^{2}\right) \\
& =\frac{|\alpha-\beta|}{\omega-|\alpha+\beta|} \operatorname{Re} t[\psi],
\end{aligned}
$$

the form $t$ is sectorial. Moreover, it is closed, since Re $t$ is closed on the given domain. The operator $H$ is then defined via the first representation theorem, 
$c f$. 44, Thm. VI.2.1], as the unique $m$-sectorial operator associated with $t$. The operator $H$ has a compact resolvent, which follows from 44, Thm. VI.3.3] and the fact that $\operatorname{Re} H$ has a compact resolvent; notice that $\operatorname{Re} H$ is an operator associated with Ret and it can be verified that

$$
\operatorname{Re} H=-(\omega+\alpha+\beta) \frac{\mathrm{d}^{2}}{\mathrm{~d} x^{2}}+(\omega-\alpha-\beta) x^{2},
$$

as expected.

- Spectrum and pseudospectrum. Since the resolvent of $H$ is compact, the spectrum of $H$ is discrete. To find the eigenvalues of $H$, we observe that $H$ is formally similar to a self-adjoint harmonic oscillator. Indeed, substituting (34) to (41) and completing the square, we find

$$
H=-(\omega-\alpha-\beta)\left(\frac{\mathrm{d}}{\mathrm{d} x}+\frac{\beta-\alpha}{\omega-\alpha-\beta} x\right)^{2}+\frac{\omega^{2}-4 \alpha \beta}{\omega-\alpha-\beta} x^{2} .
$$

From this formula it is clear that $H$ is self-adjoint if, and only if, $\alpha=\beta$. For $\alpha \neq \beta, H$ is neither self-adjoint nor $\mathcal{T}$-self-adjoint, but it is $\mathcal{P} \mathcal{T}$-symmetric and in fact real. The difference $\alpha-\beta$ acts as a sort of imaginary magnetic field. The magnetic field can be gauged out in one dimension; employing the same gauge transform for our "imaginary magnetic field," we formally check that $\tilde{H}_{\mathrm{sa}}=\Omega H \Omega^{-1}$ with

$$
\tilde{H}_{\mathrm{sa}}:=-(\omega-\alpha-\beta) \frac{\mathrm{d}^{2}}{\mathrm{~d} x^{2}}+\frac{\omega^{2}-4 \alpha \beta}{\omega-\alpha-\beta} x^{2}, \quad \Omega:=\exp \left(\frac{\beta-\alpha}{\omega-\alpha-\beta} \frac{x^{2}}{2}\right)
$$

The word "formal" refers again to the fact that $\Omega$ is unbounded. Nevertheless, the similarity relation $\tilde{H}_{\mathrm{sa}}=\Omega H \Omega^{-1}$ is well defined on eigenfunctions of $\tilde{H}_{\mathrm{sa}}$ and we can deduce that

$$
\sigma(H)=\sigma\left(\tilde{H}_{\mathrm{sa}}\right)=\left\{(2 k+1) \sqrt{\omega^{2}-4 \alpha \beta} \mid k=0,1, \ldots\right\},
$$

since eigenfunctions of $H$ are complete in $L^{2}(\mathbb{R})$. The latter can be verified by adapting the standard proof of completeness of Hermite functions; see e.g. 17. Ex. 2.2.3].

Large pseudoeigenvalues can be shown to exist by applying Theorem [ as before. Employing the unitary transform (25), we introduce a semiclassical analogue of $H$ via $\mathcal{U} H \mathcal{U}^{-1}=\sigma^{2} H_{h}$, where $H_{h}$ is (47) with the small number $h:=$ $\sigma^{-2}$ put in front of the derivative. For the symbol $f$ associated with $H_{h}$ we get

$$
\{f, \bar{f}\}=8 i(\alpha-\beta)\left[(\omega+\alpha+\beta) \xi^{2}-(\omega-\alpha-\beta) x^{2}\right] .
$$

Consequently, $\Lambda$ has the same structure as that of the rotated harmonic oscillator: a cone in the right complex half-plane with two semiaxes removed. Applying Theorem 11 we see that there is exponentially rapid resolvent growth in the interior of the cone. Again, for any $\varepsilon>0$ there are complex points with positive real part, non-zero imaginary part, and large magnitude that lie in the pseudospectrum $\sigma_{\varepsilon}(H)$.

Although the straightforward application of Theorem 1 already shows nontrivial character of the pseudospectrum, there is significantly more structure to 
be found. In fact, we show that $H$ is unitarily equivalent to a certain rotated harmonic oscillator, discussed in Section 7.4, and therefore information on the pseudospectrum and basis properties of eigenfunctions can be transferred directly to Swanson's model. Such a unitary equivalence is not a special property of this particular model; see Section 7.8

In our particular case, a suitable unitary transform for $H$ reads

$$
\begin{aligned}
\mathcal{U} & :=\mathcal{U}_{1} \mathcal{U}_{2} \mathcal{U}_{3}, \\
\left(\mathcal{U}_{1} \psi\right)(x) & :=e^{-i \delta x^{2} / 2} \psi(x), \\
\left(\mathcal{U}_{2} \psi\right)(x) & :=\left(\mathcal{F}^{-1} e^{-i \xi^{2} /(4 \delta)} \mathcal{F} \psi\right)(x), \\
\left(\mathcal{U}_{3} \psi\right)(x) & :=(2 \delta)^{1 / 4} \psi\left((2 \delta)^{1 / 2} x\right),
\end{aligned}
$$

where $\delta:=(\omega+\alpha+\beta)^{1 / 2}(\omega-\alpha-\beta)^{-1 / 2}$ and $\mathcal{F}$ is the unitary Fourier transform

$$
\mathcal{F} u(\xi)=\frac{1}{\sqrt{2 \pi}} \int_{\mathbb{R}} e^{-i x \xi} u(x) \mathrm{d} x, \quad u \in L^{1}(\mathbb{R}) \cap L^{2}(\mathbb{R}) .
$$

The key steps are the relations for $\mathfrak{U}_{2}^{*} \mathfrak{U}_{1}^{*} x \mathfrak{U}_{1} \mathfrak{U}_{2}$ and $\mathfrak{U}_{2}^{*} \mathfrak{U}_{1}^{*} p \mathfrak{U}_{1} \mathfrak{U}_{2}$. Several straightforward manipulations and properties of $\mathcal{F}$ yield that for any $\varphi \in \mathscr{S}(\mathbb{R})$

$$
\mathfrak{U}_{2}^{*} \mathfrak{U}_{1}^{*} x \mathfrak{U}_{1} \mathfrak{U}_{2} \varphi=\left(x-\frac{i}{2 \delta} \frac{\mathrm{d}}{\mathrm{d} x}\right) \varphi, \quad \mathfrak{U}_{2}^{*} \mathfrak{U}_{1}^{*} p \mathfrak{U}_{1} \mathfrak{U}_{2} \varphi=\left(-\frac{i}{2} \frac{\mathrm{d}}{\mathrm{d} x}-\delta x\right) \varphi .
$$

The latter implies

$$
u_{2}^{*} u_{1}^{*} H u_{1} u_{2}=\zeta\left(-\frac{1}{2 \delta} \frac{\mathrm{d}^{2}}{\mathrm{~d} x^{2}}+2 \delta \frac{\bar{\zeta}}{\zeta} x^{2}\right)
$$

with $\zeta:=\sqrt{\omega^{2}-(\alpha+\beta)^{2}}+i(\alpha-\beta)$. The additional rescaling $\mathcal{U}_{3}$ finally gives a multiple of the rotated harmonic oscillator:

$$
\mathcal{u}^{*} H u=\zeta\left(-\frac{\mathrm{d}^{2}}{\mathrm{~d} x^{2}}+\frac{\bar{\zeta}}{\zeta} x^{2}\right)
$$

The unitary equivalence shows that the eigenvectors of $H$ do not form a basis and the norms of the spectral projections grow as in (37) with an appropriately chosen $\theta$. Moreover, the numerically computed pseudospectra for $H$ correspond to those for the rotated oscillator in Figure 4 after the appropriate adjustment of parameters. As in previous examples, the existence of non-trivial pseudospectra makes $H$ very different from any self-adjoint operator, despite the reality of its spectrum and a formal similarity to a self-adjoint operator. In particular, the spectrum is highly unstable under small perturbations.

\subsection{Elliptic quadratic operators}

To better understand and extend the reduction (52) applied to the gauged oscillator, we now discuss general operators which are quadratic in $(x, \mathrm{~d} / \mathrm{d} x)$. We begin with a quadratic symbol $q: \mathbb{R}^{2} \rightarrow \mathbb{C}$,

$$
q(x, \xi):=\alpha x^{2}+2 \beta x \xi+\gamma \xi^{2},
$$


where the $x$ variable represents the multiplication operator and the $\xi$ variable represents the self-adjoint momentum operator $-i \mathrm{~d} / \mathrm{d} x$ defined on $W^{1,2}(\mathbb{R})$. Such a representation necessarily involves a choice for $x \xi$ between $x(\mathrm{~d} / \mathrm{d} x)$ and $(\mathrm{d} / \mathrm{d} x) x$; in the quadratic case, the Weyl quantization makes the choice

$$
x \xi \mapsto \frac{1}{2}\left(x\left(-i \frac{\mathrm{d}}{\mathrm{d} x}\right)+\left(-i \frac{\mathrm{d}}{\mathrm{d} x}\right) x\right) .
$$

This choice ensures that real-valued $q$ lead to self-adjoint operators, in addition to other convenient properties. (See, for instance, [31, Chap. 7] or [43, Sec. 18.5] for a far more general setting.)

We therefore arrive at the operator

$$
\begin{aligned}
Q & :=q^{w}(x, \xi)=\alpha x^{2}-i \beta\left(x \frac{\mathrm{d}}{\mathrm{d} x}+\frac{\mathrm{d}}{\mathrm{d} x} x\right)-\gamma \frac{\mathrm{d}^{2}}{\mathrm{~d} x^{2}}, \\
\operatorname{Dom} Q & :=\left\{u \in L^{2}(\mathbb{R}) \mid Q u \in L^{2}(\mathbb{R})\right\} .
\end{aligned}
$$

The characterization of the domain as the graph closure of the restriction to $\mathscr{S}(\mathbb{R})$ or $C_{0}^{\infty}(\mathbb{R})$ may be found in [42, pp. $\left.425-426\right]$.

- Ellipticity It is natural to assume that $q(x, \xi)$ only vanishes at the origin:

$$
q(x, \xi)=0 \Longrightarrow(x, \xi)=(0,0) .
$$

However, this is not sufficient to rule out degenerate behaviour of $Q$, and so one adds the assumption that

$$
q\left(\mathbb{R}^{2}\right) \neq \mathbb{C} .
$$

These conditions together assure us that there exists some nonzero complex number $\mu \in \mathbb{C}$ for which

$$
\operatorname{Re}(\mu q(x, \xi)) \geq|(x, \xi)|^{2}
$$

and thus $\operatorname{Re}(\mu Q)=\frac{1}{2}\left(\mu Q+\bar{\mu} Q^{*}\right)$ acts like a harmonic oscillator.

If (54) holds but (55) fails, we find ourselves in a situation resembling that of a creation or annihilation operator (34) squared: either $\operatorname{dim} \operatorname{Ker}(Q-z)=2$ for all $z \in \mathbb{C}$ or $\operatorname{dim} \operatorname{Ran}(Q-z)^{\perp}=2$ for all $z \in \mathbb{C}$; see [58, Sec. 3.1]. This is precisely the situation which arises for the gauged oscillator, Section 7.7 . when (42) holds but (44) fails.

Under (54) and (55), the spectral theory of the operator $Q$ can be deduced from the spectral theory of the matrix sometimes called the fundamental matrix:

$$
F:=\left(\begin{array}{cc}
\beta & \gamma \\
-\alpha & -\beta
\end{array}\right) .
$$

It is shown in [73, Prop. 3.3] that

$$
\begin{aligned}
\sigma(F) & = \pm \lambda, \\
\operatorname{Ker}(F \mp \lambda) & =\operatorname{span}\left\{\left(1, a_{ \pm}\right)\right\}, \quad \pm \operatorname{Im} a_{ \pm}>0 .
\end{aligned}
$$

We note that, in dimension 1 , we can identify $\mu=i / \lambda$ in (56) through choosing $\lambda$ according to the signs of $\operatorname{Im} a_{ \pm}$. Taking this notation into account, we then have from [73, Thm. 3.5] that

$$
\sigma(Q)=\{-i \lambda(2 k+1) \mid k=0,1,2, \ldots\} .
$$


- Linear symplectic transformations One advantage of the Weyl quantization is that we may transform our symbols by composition with symplectic transformations; see e.g. [43, Sec. 18.5] for a detailed accounting of the theory. In our simplified (linear, dimension one) setting, the set of real linear symplectic transformations is simply the set of a 2 -by-2 matrices with real entries and determinant equal to one. Any such matrix may be written [43, Lem. 18.5.8] as a composition of matrices of the form

$$
G_{b}:=\left(\begin{array}{ll}
1 & 0 \\
b & 1
\end{array}\right), \quad V_{c}:=\left(\begin{array}{cc}
c & 0 \\
0 & 1 / c
\end{array}\right), \quad J:=\left(\begin{array}{cc}
0 & 1 \\
-1 & 0
\end{array}\right) .
$$

with $b, c \in \mathbb{R}$ and $c \neq 0$.

What is more, one may transform a quadratic symbol by composing with such a matrix by conjugating with an easily-understood unitary transformation on $L^{2}(\mathbb{R})$. Specifically, we use multiplication by a complex Gaussian

$$
\mathcal{G}_{b} u(x):=e^{i b x^{2} / 2} u(x),
$$

a scaling change of variables,

$$
\mathcal{V}_{c} u(x):=c^{-1 / 2} u(x / c),
$$

and the unitary Fourier transform (49). Using definition (53), it is then straightforward to check that

$$
\begin{aligned}
\mathcal{G}_{b}^{*} q^{w}(x, \xi) \mathcal{G}_{b} & =\left(q \circ G_{b}\right)^{w}(x, \xi), \\
\mathcal{V}_{c}^{*} q^{w}(x, \xi) \mathcal{V}_{c} & =\left(q \circ V_{c}\right)^{w}(x, \xi), \\
\mathcal{F}^{*} q^{w}(x, \xi) \mathcal{F} & =(q \circ J)^{w}(x, \xi) .
\end{aligned}
$$

One important example is the change of variables which gives us the correspondence between high-energy and semiclassical limits: for any $z \in \mathbb{C}$,

$$
q^{w}(x, \xi)-z=\mathcal{V}_{\sqrt{h}}^{*}\left(\frac{1}{h}\left(q^{w}(x, h \xi)-h z\right)\right) \mathcal{V}_{\sqrt{h}} .
$$

Therefore the regime with spectral parameter $r z$ as $r \rightarrow \infty$ for the operator $q^{w}(x, \xi)$ is unitarily equivalent to the regime with spectral parameter $z$ fixed for the operator $\frac{1}{h} q^{w}(x, h \xi)$ as $h=1 / r \rightarrow 0^{+}$.

- Reduction to rotated harmonic oscillator In [57, Lem. 2.1], PravdaStarov identifies a procedure for taking an elliptic quadratic form $q$ and finding $\mu \in \mathbb{C} \backslash\{0\}$ and a real matrix $T$ with $\operatorname{det} T=1$ for which

$$
(q \circ T)(x, \xi)=\mu\left(\left(1+i \lambda_{1}\right) x^{2}+\left(1+i \lambda_{2}\right) \xi^{2}\right)
$$

for $\lambda_{1}, \lambda_{2} \in \mathbb{R}$. Applying a scaling like (62) and scaling $\mu$ allows us to assume that the coefficients of $x^{2}$ and $\xi^{2}$ have the same modulus. It is then evident that the resulting symbol is a multiple of that of a rotated harmonic oscillator (35).

If one wishes only to identify the parameters of the rotated harmonic oscillator involved, one may appeal to the spectral decomposition of the fundamental 
matrix and the growth of the spectral projections. An application of Corollary 1.7 in 79 in terms of the eigensystem (58) shows that the norm of the spectral projections $P_{k}$ for the eigenvalues $(2 k+1) \lambda / i$ obey the asymptotics

$$
\lim _{k \rightarrow \infty} \frac{\log \left\|P_{k}\right\|}{k}=\frac{1}{2} \log \frac{1+\left|c_{+}\right|}{1-\left|c_{+}\right|}, \quad c_{+}=-\frac{a_{+}-\overline{a_{-}}}{a_{+}-a_{-}} .
$$

From (37), we see that this uniquely identifies a rotated harmonic oscillator with $\theta \geq 0$. The multiplicative factor, in turn, is determined by the ground state energy, that is, the eigenvalue corresponding to $k=0$. We arrive at the following proposition.

Proposition 8. Let $Q$ be any quadratic operator as in (53) with symbol $q$. Assume that $q$ is elliptic as in (54) and (55), and therefore let the eigensystem of the fundamental matrix of $q$ be as in (58). Let $\theta \in[0, \pi / 2)$ be determined by

$$
\sin \theta=\left|\frac{a_{+}-\overline{a_{-}}}{a_{+}-a_{-}}\right| .
$$

Then $Q$, as an unbounded operator on $L^{2}(\mathbb{R})$, is unitarily equivalent to

$$
\lambda\left(-e^{-i \theta} \frac{\mathrm{d}^{2}}{\mathrm{~d} x^{2}}+e^{i \theta} x^{2}\right) .
$$

- Higher dimension The extension of the spectral and pseudospectral theory to elliptic quadratic operators in higher dimension is well-developed but not complete. We content ourselves with a brief description and references.

The Weyl quantization of a quadratic form

$$
q(x, \xi): \mathbb{R}^{2 d} \rightarrow \mathbb{C}
$$

in dimension $d \geq 2$ also associates the variable $x_{j}$ with multiplication by $x_{j}$, associates the variable $\xi_{j}$ with $-i \partial / \partial x_{j}$, and resolves the problem of commutativity (in the quadratic case) by taking an average:

$$
\left(x_{j} \xi_{j}\right)^{w} u(x)=\frac{1}{2 i}\left(x_{j} \frac{\partial}{\partial x_{j}} u(x)+\frac{\partial}{\partial x_{j}}\left(x_{j} u(x)\right)\right) .
$$

The ellipticity hypothesis is simpler, since in dimension 2 or greater, (54) implies (55), shown in [73, Lem. 3.1].

Under the assumption (54), the spectrum of $Q=q^{w}(x, \xi)$ is a lattice determined by eigenvalues of the matrix corresponding to the fundamental matrix (57); the formula is given in [73, Thm. 3.5].

It was recently shown in [21] that, regardless of dimension, an elliptic quadratic form obeying a $\mathcal{P T}$-symmetry condition is formally similiar to a self-adjoint operator if and only if the spectrum is real and the fundamental matrix is diagonalizable. This similarity is only enacted eigenspace by eigenspace, and the authors observe that the pseudospectral considerations prevent the similarity transformations from being bounded with bounded inverse on $L^{2}\left(\mathbb{R}^{d}\right)$.

In 58, Pravda-Starov conducts a complete study of the semiclassical pseudospectrum for non-normal elliptic quadratic operators. It is shown in 58, 
Sec. 3.2] that, for a non-normal operator, the bracket condition is violated everywhere in the interior of the range of the symbol and therefore exponentially accurate pseudomodes exist.

Conversely, exponential upper bounds for the resolvent are proven in [41, except that exponential growth $C^{1 / h}$ may need to be replaced by the more rapid growth $(C / h)^{C / h}$ when the fundamental matrix contains Jordan blocks.

Finally, the associated spectral projections for a non-normal quadratic operator were shown in 79 to usually increase at an exponential rate, though there are degenerate situations such as when Jordan blocks are present in the fundamental matrix.

\subsection{Numerical computation of JWKB solutions}

The proof of Theorem 1 proceeds by creating pseudomodes as JWKB (JeffreysWentzel-Kramers-Brillouin) approximations for which $(H-z) u \approx 0$. Focusing on the one-dimensional Schrödinger case, these functions can be expressed using a few elementary operations, including differentiation and integration; see (67), (70), (72), and (73). The software package Chebfun [78, allows us to easily compute these pseudomodes with high accuracy.

In Figure 7 we compare a JWKB pseudomode for the semiclassical rotated harmonic oscillator

$$
H_{h}=-h^{2} e^{-i \pi / 4} \frac{\mathrm{d}^{2}}{\mathrm{~d} x^{2}}+e^{i \pi / 4} x^{2}
$$

discussed in Section 7.4 with $z=e^{-i \pi / 4}(1 / 2+i), h=2^{-5}$, and

$$
u(x ; h)=\chi(x) e^{i \varphi(x) / h} \sum_{j=0}^{6} h^{j} a_{j}(x) .
$$

The plot on the left is of the real part (red) and the imaginary part (blue) of the pseudomode, and the plot on the right is of the image, $\left(H_{h}-z\right) u$. One may compute that

$$
\frac{\left\|\left(H_{h}-z\right) u\right\|}{\|u\|} \approx 2.5041 \times 10^{-4} .
$$

One can clearly see the contributions from the gradient of the support of the cutoff function, which is $[0.2,0.4] \cup[1.6,1.8]$.

If one studies instead the semiclassical rescaling of the shifted harmonic oscillator

$$
H_{h}=-h^{2} \frac{\mathrm{d}^{2}}{\mathrm{~d} x^{2}}+x^{2}+2 i h^{1 / 2} x-h
$$

discussed in Section 7.5 we have noticeable but less accurate pseudomodes, with the principal error given on the support of the cutoff function. In Figure 8 , one has a similar JWKB solution and its image with $h=2^{-8}, z=2-h+2 i h^{1 / 2}$, and

$$
u(x ; h)=\chi(x) e^{i \varphi(x) / h} \sum_{j=0}^{2} h^{j} a_{j}(x) .
$$

(We may see numerically that there is practically no difference in norms when taking one, two, or ten terms in the expansion.) Since $h$ is very small, the JWKB function oscillates quite rapidly, and since the decay of $e^{i \varphi / h}$ is comparatively 

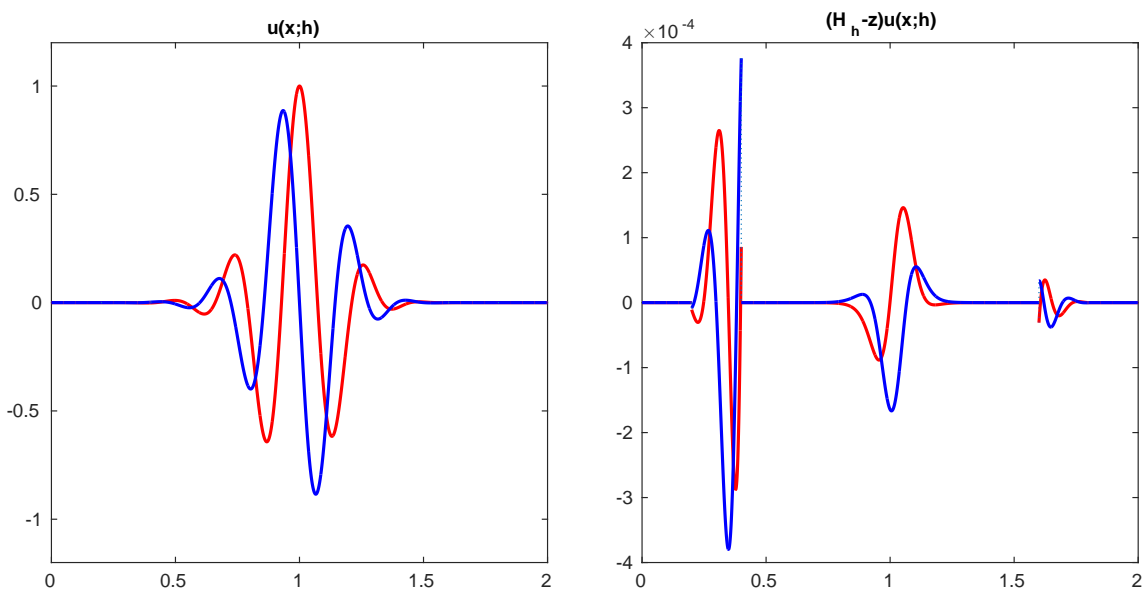

Figure 7: Pseudomode (left) and image (right) for semiclassical rotated harmonic oscillator. The red curve is the real part, and the blue the imaginary.

weak, the principal error comes from the cutoff function, whose gradient is again supported on $[0.2,0.4] \cup[1.6,1.8]$. We have here

$$
\frac{\left\|\left(H_{h}-z\right) u\right\|}{\|u\|} \approx 2.0290 \times 10^{-3} .
$$
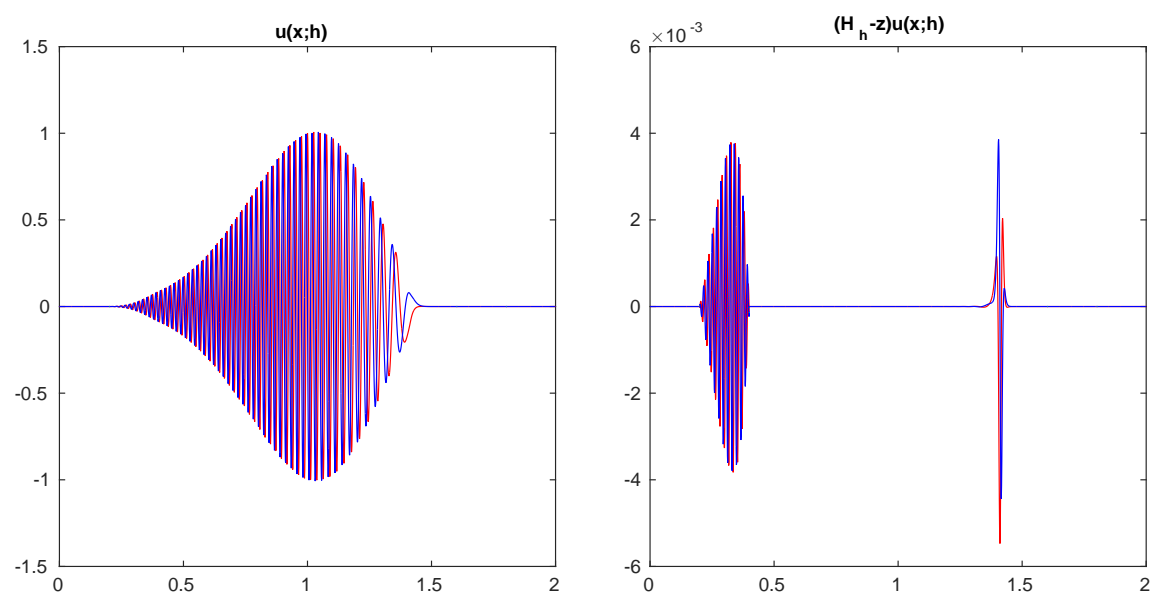

Figure 8: Pseudomode (left) and image (right) for semiclassical rescaling of shifted harmonic oscillator. The red curve is the real part, and the blue the imaginary.

We can then compare the accuracy of the $L^{2}(\mathbb{R})$-normalized pseudomodes by plotting $\left\|\left(H_{h}-z\right) u\right\|$ versus $1 / h$ for a variety of $h$, presented in Figure 9 . On the left, we have the norms for the semiclassical rotated harmonic oscillator (64) 
at $z=1+4 i$, and on the right, we have those for the rescaled shifted harmonic oscillator (65) at $z=2-h+2 i h^{1 / 2}$. We can observe that the norm ratios for the pseudomodes for the rotated harmonic oscillator decrease like $\exp (-c / h)$ while those for the shifted harmonic oscillator decrease more slowly.
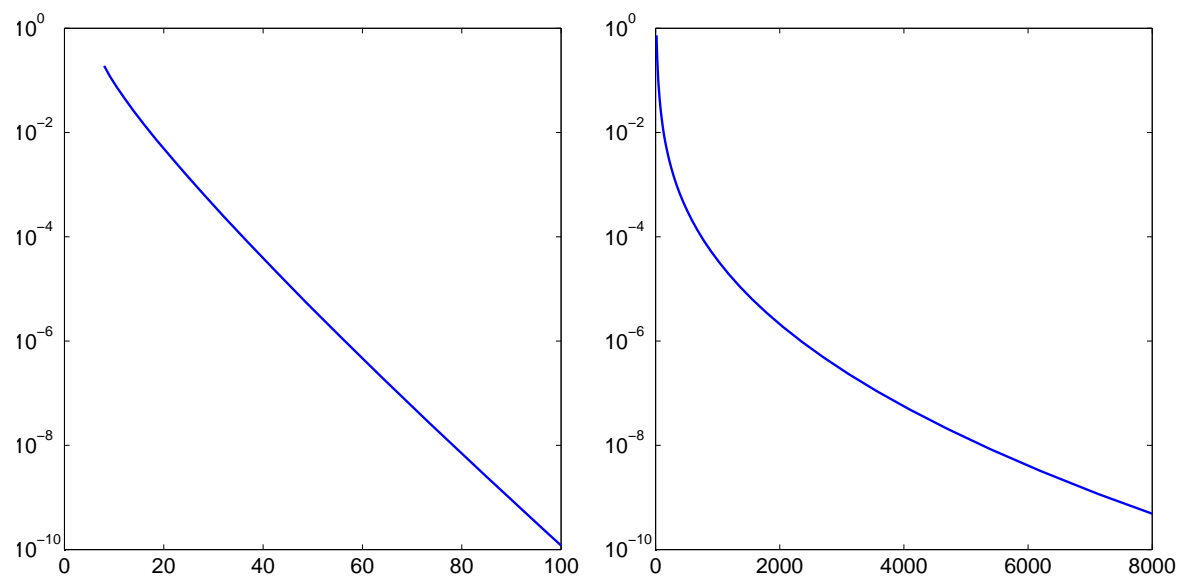

Figure 9: $\left\|\left(H_{h}-z\right) u\right\|$ as a function of $1 / h$ for normalized pseudomodes for rotated harmonic oscillator (left) and shifted harmonic oscillator (right).

\section{A Existence proofs for pseudomodes}

For the interested reader, we include detailed proofs of Theorem 1 in the case of a Schrödinger operator and of Theorem 7 . In these proofs, the constant $C>0$ may change from line to line. Furthermore, we understand semiclassical statements involving $h$ to only hold for $h \in\left(0, h_{0}\right]$ for some $h_{0}>0$; so long as $h_{0}$ changes only finitely many times in the proof, we are allowed to make conclusions "for $h$ sufficiently small" in our theorems.

\section{A.1 Proof of special case of Theorem 1}

We restrict our attention to the case

$$
H_{h}=-h^{2} \frac{\mathrm{d}^{2}}{\mathrm{~d} x^{2}}+V(x)
$$

The symbol of $H_{h}$ is

$$
f(x, \xi)=\xi^{2}+V(x)
$$

and

$$
\frac{1}{2 i}\{f, \bar{f}\}=-2 \xi \operatorname{Im} V^{\prime}(x) .
$$

Therefore $z \in \Lambda$, defined in (15), if and only if there exists $\left(x_{0}, \xi_{0}\right) \in \mathbb{R}^{2}$ with $z=\xi_{0}^{2}+V\left(x_{0}\right), \operatorname{Im} V^{\prime}\left(x_{0}\right) \neq 0$, and $-\xi_{0} \operatorname{Im} V^{\prime}\left(x_{0}\right)>0$. Equivalently, since we may choose the sign of $\xi_{0}$, there exists some $x_{0} \in \mathbb{R}$ where $\operatorname{Im} V^{\prime}\left(x_{0}\right) \neq 0$ 
and $z-V\left(x_{0}\right)$ is a positive real number. We only need to assume that $V(x)$ is analytic in a neighborhood of $x_{0}$.

After a translation, we may assume that $x_{0}=0$. We seek a JWKB approximation (see for instance [31, Chap. 2]) to a solution of $\left(H_{h}-z\right) u=0$ of the form

$$
u(x ; h)=e^{i \varphi(x) / h} \sum_{j=0}^{N(h)} h^{j} a_{j}(x)
$$

for $a_{j}(x)$ analytic near $x_{0}=0$. The strategy is to choose the phase function such that conjugation by the multiplication operator $e^{-i \varphi(x) / h}$ reduces $H_{h}$ to a transport equation plus an error in $h$. The functions $a_{j}$ may be found iteratively and then $N(h)$ may be chosen to give an accurate local solution. The quasimode will then be obtained by multiplying $u(x ; h)$ by a fixed cutoff function $\chi$ localizing to a neighborhood of $x_{0}=0$. An important difference making the JWKB theory for non-self-adjoint operators somewhat simpler is that the phase function $\varphi(x)$ has a significant imaginary part. This allows for multiplication by cutoff functions with small errors, a technique which is generally not available for self-adjoint operators where $\varphi$ is real-valued.

We require that the phase function $\varphi(x)$ satisfies the eikonal equation

$$
f\left(x, \varphi^{\prime}(x)\right)-z=0
$$

for $f$ from (66). Clearly this implies that $\varphi^{\prime}(x)= \pm \sqrt{z-V(x)}$. Since $z-V(0)>$ 0 , this function is analytic in a neighborhood of $0 \in \mathbb{C}$.

We allow the sign to be determined by the bracket condition

$$
\frac{1}{2 i}\{f, \bar{f}\}(x, \xi)=-2 \operatorname{Im} V^{\prime}(x) \xi>0 .
$$

Applying this to $(x, \xi)=\left(0, \varphi^{\prime}(0)\right)$ indicates that the sign of $\varphi^{\prime}(0)$ should be taken to be the opposite of the sign of $\operatorname{Im} V^{\prime}(0)$. Alternately, the importance of this choice of sign may be seen by observing that

$$
\varphi^{\prime \prime}(x)=-\frac{V^{\prime}(x)}{2 \varphi^{\prime}(x)}
$$

and thus our choice is made so that $\operatorname{Im} \varphi^{\prime \prime}(0)>0$, which means that $e^{i \varphi(x) / h}$ has rapid decay away from $x=0$. We arrive at the formula

$$
\varphi(x)=-\operatorname{sgn}\left(\operatorname{Im} V^{\prime}\left(x_{0}\right)\right) \int_{0}^{x} \sqrt{z-V(y)} \mathrm{d} y .
$$

We may then check that

$$
e^{-i \varphi / h}\left(H_{h}-z\right) e^{i \varphi / h}=\frac{2 h}{i}\left(\varphi^{\prime} \frac{\mathrm{d}}{\mathrm{d} x}+\frac{1}{2} \varphi^{\prime \prime}\right)-h^{2} \frac{\mathrm{d}^{2}}{\mathrm{~d} x^{2}} .
$$

So long as $\left\{a_{j}\right\}_{j=0}^{\infty}$ satisfy the transport equations

$$
\varphi^{\prime}(x) a_{0}^{\prime}(x)+\frac{1}{2} \varphi^{\prime \prime}(x) a_{0}(x)=0
$$

and

$$
\varphi^{\prime}(x) a_{j}^{\prime}(x)+\frac{1}{2} \varphi^{\prime \prime}(x) a_{j}(x)=\frac{i}{2} a_{j-1}^{\prime \prime}(x), \quad j=1,2, \ldots,
$$


we have

$$
e^{-i \varphi / h}\left(H_{h}-z\right) e^{i \varphi / h}\left(\sum_{j=0}^{N} h^{j} a_{j}\right)=-h^{N+2} a_{N}^{\prime \prime} .
$$

We are free to choose $a_{0}(0)=1$ and $a_{j}(0)=0$ for all $j>0$. Using the integrating factor $\exp \left(\int_{0}^{x} \varphi^{\prime \prime}(y) /\left(2 \varphi^{\prime}(y)\right) \mathrm{d} y\right)=C \sqrt{\varphi^{\prime}(x)}$ immediately gives that

$$
a_{0}(x)=\frac{\sqrt{\varphi^{\prime}(0)}}{\sqrt{\varphi^{\prime}(x)}}
$$

and that, for $j>0$,

$$
a_{j+1}(x)=\frac{1}{\sqrt{\varphi^{\prime}(x)}} \int_{0}^{x} \frac{i a_{j}^{\prime \prime}(y)}{2 \sqrt{\varphi^{\prime}(y)}} d y .
$$

We note that, in a sufficiently small neighborhood of zero in the complex plane, $\varphi^{\prime}$ may be extended to an analytic function which is bounded away from zero, and therefore each $a_{j}$ is certainly analytic on that neighborhood of zero.

We now consider bounds on the functions $a_{j}$. As in Example 1.1 of [74], we will show that the $a_{j}$ obey the estimates

$$
\left|a_{j}(z)\right| \leq C_{1}^{j+1} j^{j}
$$

for some $C_{1}>0$ and all $z$ in a neighborhood of the origin. A sequence of functions satisfying these estimates is said to be a formal analytic symbol. Once these bounds are established, we may define the $h$-dependent function

$$
a(z ; h)=\sum_{0 \leq j \leq\left(e C_{1} h\right)^{-1}} h^{j} a_{j}(z),
$$

summing over a collection of $j$ chosen such that

$$
\left|h^{j} a_{j}(z)\right| \leq C_{1}\left(C_{1} h j\right)^{j} \leq C_{1} e^{-j} .
$$

Since $\left\{e^{-j}\right\}_{j \geq 0}$ is summable, we will therefore have that $\{a(z ; h)\}_{0<h<h_{0}}$ is a uniformly bounded collection of analytic functions on the set where (74) holds.

The natural norm to use here for analytic functions is the supremum norm, so for $K \subseteq \mathbb{C}$ we write

$$
\|g\|_{K}=\sup _{z \in K}|g(z)| .
$$

For balls in the complex plane centered at zero, we use the notation

$$
B(R)=\{z \in \mathbb{C}|| z \mid<R\} .
$$

Fix $R_{0}>0$ such that, on $B\left(R_{0}\right)$, the phase function $\varphi$ is analytic, the modulus of the derivative $\left|\varphi^{\prime}\right|$ is bounded from above and below, and $\operatorname{Im} \varphi^{\prime \prime}(x)>1 / C$ for some $C>0$.

Cauchy's estimates for the second derivative of a analytic bounded function $g$ defined on $B(R)$ read

$$
\left|g^{\prime \prime}(z)\right| \leq \frac{2\|g\|_{B(R)}}{(R-|z|)^{2}} .
$$


We integrate the estimates applied to $a_{j}^{\prime \prime}$ to obtain bounds for $a_{j+1}$ :

$$
\begin{aligned}
\left|a_{j+1}(z)\right| & =\left|\frac{1}{\sqrt{\varphi^{\prime}(z)}} \int_{0}^{z} \frac{i a_{j}^{\prime \prime}(\zeta)}{2 \sqrt{\varphi^{\prime}(\zeta)}} d \zeta\right| \\
& \leq\left\|\left(\varphi^{\prime}\right)^{-1}\right\|_{B(R)} \int_{0}^{|z|} \frac{\left\|a_{j}\right\|_{B(R)}}{(R-t)^{2}} \mathrm{~d} t \\
& \leq\left\|\left(\varphi^{\prime}\right)^{-1}\right\|_{B(R)}\left\|a_{j}\right\|_{B(R)}\left(\frac{1}{R-|z|}-\frac{1}{R}\right) \\
& =\frac{|z|}{R(R-|z|)}\left\|\left(\varphi^{\prime}\right)^{-1}\right\|_{B(R)}\left\|a_{j}\right\|_{B(R)} .
\end{aligned}
$$

The estimate for $\left|a_{j+1}(z)\right|$ is stronger than the usual Cauchy's estimate for the first derivative when $z$ is near zero, which corresponds to having set $a_{j+1}(0)=0$.

To obtain the estimates (74) on $B\left(R_{0} / 2\right)$, we fix $j>0$ and iterate (78) on balls of radius

$$
R_{k}=\left(1-\frac{k}{2 j}\right) R_{0}, \quad k=0, \ldots, j-1
$$

When $|z| \leq R_{k+1}$, we have

$$
\frac{|z|}{R_{k}\left(R_{k}-|z|\right)} \leq \frac{|z|}{R_{k}\left(R_{k}-R_{k+1}\right)}=\frac{|z|}{\left(R_{0} / 2\right)\left(R_{0} / 2 j\right)} \leq \frac{4 j|z|}{R_{0}^{2}},
$$

since $R_{k}>R_{0} / 2$ when $k<j$. Therefore we may bound $a_{k+1}$ on the disc of radius $R_{k+1}$ using a bound for $a_{k}$ on the disc of radius $R_{k}$ and (78):

$$
\begin{aligned}
\left\|a_{k+1}\right\|_{B\left(R_{k+1}\right)} & \leq \frac{|z|}{R_{k}\left(R_{k}-|z|\right)}\left\|\left(\varphi^{\prime}\right)^{-1}\right\|_{B\left(R_{k}\right)}\left\|a_{k}\right\|_{B\left(R_{k}\right)} \\
& \leq \frac{4 j|z|}{R_{0}^{2}}\left\|\left(\varphi^{\prime}\right)^{-1}\right\|_{B\left(R_{0}\right)}\left\|a_{k}\right\|_{B\left(R_{k}\right)} .
\end{aligned}
$$

Therefore, when $j>0$, we take the product of the estimates (79) for $k=$ $0, \ldots, j-1$ to obtain

$$
\left\|a_{j}\right\|_{B\left(R_{0} / 2\right)} \leq\left\|a_{0}\right\|_{B\left(R_{0}\right)}\left(C_{2}|z| j\right)^{j}, \quad C_{2}=\frac{4}{R_{0}^{2}}\left\|\left(\varphi^{\prime}\right)^{-1}\right\|_{B\left(R_{0}\right)} .
$$

The estimate (74) on $B\left(R_{0} / 2\right)$ immediately follows, with

$$
C_{1}=\max \left(\left\|a_{0}\right\|_{B\left(R_{0}\right)}, \frac{2}{R_{0}}\left\|\left(\varphi^{\prime}\right)^{-1}\right\|_{B\left(R_{0}\right)}\right) .
$$

Having established estimates for $a(z ; h)$ when $z \in B\left(R_{0} / 2\right)$, let $\chi \in C_{0}^{\infty}(\mathbb{R})$ be equal to one in a neighborhood of $0 \in \mathbb{R}$ and have support in a compact subset of the interval $\left(-R_{0} / 2, R_{0} / 2\right)$. We then define our pseudomode as

$$
u(x ; h)=e^{i \varphi(x) / h} \chi(x) a(x ; h),
$$

with $a(x ; h)$ defined in 75 .

We then estimate the $L^{2}(\mathbb{R})$ norm

$$
\left.\| H_{h}-z\right) u(x ; h)\|\leq\| \chi\left(H_{h}-z\right) e^{i \varphi / h} a\|+\|\left[\left(H_{h}-z\right), \chi\right] e^{i \varphi / h} a \|
$$


as follows. First, we recall that we have chosen $R_{0}$ such that $\operatorname{Im} \varphi^{\prime \prime}(x)>1 / C_{3}$ for some $C_{3}>0$. Since $\varphi(0)=0$ and $\varphi^{\prime}(0)$ is real, we therefore have that

$$
\left|e^{i \varphi(x) / h}\right| \leq \exp \left(-\frac{1}{2 C_{3} h} x^{2}\right), \quad \forall x \in \operatorname{supp} \chi .
$$

Since $\left|e^{-i \varphi / h}\right| \geq 1$ on $\operatorname{supp} \chi$, we may multiply by $e^{-i \varphi / h}$ and use (71) to obtain

$$
\left\|\chi H_{h} e^{i \varphi / h} a\right\| \leq\left\|\chi e^{-i \varphi / h} H_{h} e^{i \varphi / h} a\right\|=\left\|h^{N+2} a_{N}^{\prime \prime}(x) \chi(x)\right\|,
$$

with $N=N(h)=\left\lfloor(e C h)^{-1}\right\rfloor$. Cauchy's estimates (77) along with (76) show that $h^{N+2} a_{N}^{\prime \prime}(x) \leq C e^{-1 /(C h)}$ for $C>0$ independent of $h$ and all $x \in \operatorname{supp} \chi$. We therefore have, for some $C>0$, the estimate

$$
\left\|\chi H_{h} e^{i \varphi / h} a\right\| \leq C e^{-1 /(C h)} .
$$

In the commutator of $\left(H_{h}-z\right)$ and $\chi$, only the derivatives in $H_{h}$ play a role. We compute

$$
\begin{aligned}
{\left[\left(H_{h}-z\right), \chi\right] e^{i \varphi / h} a } & =\left[-h^{2} \frac{\mathrm{d}^{2}}{\mathrm{~d} x^{2}}, \chi\right] e^{i \varphi / h} a \\
& =-h^{2} e^{i \varphi / h}\left(\chi^{\prime \prime} a+2 \chi^{\prime}\left(a^{\prime}+\frac{i \varphi^{\prime}}{h} a\right)\right) .
\end{aligned}
$$

On $\operatorname{supp}(\chi)$, we have uniform bounds on $a$ by (76) and therefore on $a^{\prime}$ by Cauchy's estimates. As before, $\varphi^{\prime}$ is controlled by the choice of $R_{0}$. Exponential decay comes from the fact that $\operatorname{supp}\left(\chi^{\prime}\right)$ avoids a neighborhood of 0 : by (83), we have that

$$
\left|e^{i \varphi(x) / h}\right| \leq e^{-1 /(C h)}, \quad \forall x \in \operatorname{supp}\left(\chi^{\prime}\right) .
$$

Therefore the second term in (82) is also exponentially small in $1 / h$.

Having proven that both terms in (82) are exponentially small, the proof is complete upon showing that $u(x ; h)$ is not exponentially small. Intuitively, this is clear from the choice of $\varphi$ and that $a_{0}(0)=1$ and $a_{j}(0)=0$ for $j>0$, from which we know that $u(x ; h)$ resembles $e^{-\varphi^{\prime \prime}(0) x^{2} /(2 h)}$ in a small neighborhood of zero. Formally, since (80) gives $\left|h^{j} a_{j}(z)\right| \leq C(C|z|)^{j}$ for $|z|<R_{0} / 2$ and $0<j \leq N(h)=\left(e C_{1} h\right)^{-1}$, we have for some $r_{0}>0$ sufficiently small the estimate

$$
\left\|\sum_{j=1}^{N(h)} h^{j} a_{j}(z)\right\|_{B(r)} \leq C r, \quad 0<r \leq r_{0} .
$$

Since $a_{0}(z)$ is close to 1 and $\operatorname{Im} \varphi(z)$ is close to $\frac{1}{2} \operatorname{Im} \varphi^{\prime \prime}(0) z^{2}$ when $z$ is close to 0 , we can consider $r$ sufficiently small and fixed to obtain

$$
\|u(x ; h)\| \geq\|u(x ; h)\|_{L^{2}((-r, r))} \geq \frac{1}{C}\left(\int_{-r}^{r} \exp \left(\frac{1}{C h} x^{2}\right) d x\right)^{1 / 2} \geq \frac{1}{C} h^{1 / 4}
$$

when $h$ is sufficiently small.

Since we have shown that $\left\|H_{h} u(x ; h)\right\| \leq C e^{-1 /(C h)}$ and $\|u(x ; h)\| \geq h^{1 / 4} / C$, this completes the proof of the theorem in this special case. 
- Uniformity on compact sets. We also remark that the exponential resolvent growth may generally be made uniform on compact subsets of $\Lambda$, the interior of the semiclassical pseudospectrum defined in (15). In the case of the Schrödinger operator, for any $z \in \Lambda$ we may take $x_{0}$ with $\operatorname{Im} V\left(x_{0}\right)=\operatorname{Im} z$ and define the phase function

$$
\varphi(x)= \pm \int_{x_{0}}^{x} \sqrt{z-V(y)} \mathrm{d} y,
$$

with the sign chosen so that $\operatorname{Im} \varphi^{\prime \prime}\left(x_{0}\right)>0$.

The exponentially rapid resolvent growth then follows from having $C>0$ and $R_{1}>R_{0}>0$ for which the estimates

$$
\left|x-x_{0}\right|<R_{1} \Longrightarrow \frac{1}{C} \leq\left|\varphi^{\prime}(x)\right| \leq C
$$

and

$$
R_{0}<\left|x-x_{0}\right|<R_{1} \Longrightarrow \operatorname{Im} \varphi(x) \geq \frac{1}{C}
$$

hold: the former gives (744) by way of (81) and the latter gives (85), which are together sufficient to prove exponential growth of the resolvent.

The condition $\operatorname{Im} V^{\prime}\left(x_{0}\right) \neq 0$ means that $x_{0}$ may be chosen locally as a continuous function of $\operatorname{Im} z$; it is then a simple matter to verify that (86) holds with uniform constants in a neighborhood of $z \in \Lambda$. Local uniformity of (87) then follows from $\operatorname{Im} \varphi^{\prime \prime}\left(x_{0}\right)>0$.

\section{A.2 Proof of Theorem 17}

As usual, we may make the change of variables $y=h^{-1 / 2} x$, arriving at an operator unitarily equivalent to $h H_{h}$ :

$$
\tilde{H}_{h}=-h^{2} \frac{\mathrm{d}^{2}}{\mathrm{~d} y^{2}}+y^{2}+2 i h^{1 / 2} y-h \simeq h H_{h} .
$$

We will let $h^{-1}=\operatorname{Re} z$ so that

$$
H_{h}-z \simeq h^{-1}\left(\tilde{H}_{h}-(1+i t)\right), \quad t=\frac{\operatorname{Im} z}{\operatorname{Re} z} .
$$

In this case, we have a symbol

$$
\tilde{f}(y, \eta)=\eta^{2}+y^{2}+2 i h^{1 / 2} y-h
$$

and so we cannot directly apply the results of [29]. We can, however, adapt the previous proof.

We can see that $1+i t=\tilde{f}\left(y_{0}, \eta_{0}\right)$ for some $\left(y_{0}, \eta_{0}\right) \in \mathbb{R}^{2}$ if and only if

$$
1+h=\eta_{0}^{2}+y_{0}^{2}, \quad t=2 i h^{1 / 2} y_{0},
$$

implying that $\left|y_{0}\right| \leq 1+h$ and

$$
|t| \leq 2 h^{1 / 2}(1+h) .
$$


The eikonal equation $\tilde{f}\left(y, \varphi^{\prime}(y)\right)=0$ is then solved by integrating

$$
\begin{aligned}
\varphi^{\prime}(y) & =-\sqrt{(1+h+i t)-i h^{1 / 2} y-y^{2}} \\
& =-\left(1+h-y^{2}\right)\left(1+\frac{i\left(t-2 h^{1 / 2} y\right)}{1+h-y^{2}}+\mathcal{O}\left(\left(\frac{t-2 h^{1 / 2} y}{1+h-y^{2}}\right)^{2}\right)\right) .
\end{aligned}
$$

As usual, we choose the sign of the square root to satisfy a bracket condition like (68).

To ensure that, on a uniform neighborhood of $y_{0}$, the phase $\varphi^{\prime}(y)$ is analytic and there exists $C>0$ for which $1 / C \leq\left|\varphi^{\prime}(y)\right| \leq C$, we must assume that $1+h-y^{2}$ is bounded away from zero by a constant. We therefore assume that $\left|y_{0}\right|<1-\varepsilon$ for some $\varepsilon>0$. We remark that this is connected to assuming that $1+i t$ is in the interior of the range of the symbol. The scaling argument shows that this assumption is equivalent to the hypothesis that

$$
\operatorname{Im} z \leq 2\left(1-\varepsilon+(\operatorname{Re} z)^{-1}\right) \sqrt{\operatorname{Re} z} .
$$

The term $(\operatorname{Re} z)^{-1}$ is negligible as $\operatorname{Re} z \rightarrow \infty$.

Note that

$$
\varphi^{\prime \prime}(y)=\frac{-i h^{1 / 2}-y}{\varphi^{\prime}(y)}
$$

and so, since we chose the sign of the square root to have $\varphi^{\prime}\left(y_{0}\right)<0$, we have

$$
\frac{1}{C} \sqrt{h} \leq \operatorname{Im} \varphi^{\prime \prime}(y) \leq C \sqrt{h}
$$

on a sufficiently small but fixed neighborhood.

We may then construct exponentially accurate approximations to a solution of

$$
e^{-i \varphi / h} \tilde{H}_{h} e^{i \varphi / h} a(x ; h)=0
$$

exactly as in the case of the semiclassical Schrödinger operator above. Taking a cutoff function $\chi$ supported near $y_{0}$ and writing

$$
u(y ; h)=\chi(y) e^{i \varphi(y) / h} a(y ; h)
$$

as before, we have the same argument for exponential smallness except where we commute $\tilde{H}_{h}$ past the cutoff function $\chi$. Because $\operatorname{Im} \varphi(y)$ increases more slowly, we only have

$$
\left|e^{i \varphi(y) / h}\right| \leq e^{-1 /\left(C h^{1 / 2}\right)}, \quad y \in \operatorname{supp}\left(\chi^{\prime}\right) .
$$

We arrive at

$$
\left\|\tilde{H}_{h} u(y ; h)\right\|_{L^{2}} \leq C e^{-1 /\left(C h^{1 / 2}\right)}
$$

and

$$
\|u(y ; h)\|_{L^{2}} \geq \frac{1}{C} h^{1 / 8}
$$

for $h$ sufficiently small and positive. Conjugating by the change of variables $y=$ $(\operatorname{Re} z)^{1 / 2} x$ with which we began and ignoring harmless powers of $h=(\operatorname{Re} z)^{-1}$ proves the theorem. 


\section{B Spectral projections of the rotated oscillator}

Asymptotics for the norms of the spectral projections of the rotated oscillator may be found using an established integral formula involving the Hermite functions, pointed out in [13, and asymptotics of the Legendre polynomials. This approach is analogous to one applied to the shifted harmonic oscillator in [51, Sec. 2], and it simplifies and sharpens the result of [27.

The eigenfunctions of $H$ defined in (35) can be written explicitly through a complex scaling of Hermite functions:

$$
H\left(h_{k}\left(e^{i \theta / 2} x\right)\right)=(2 k+1) h_{k}\left(e^{i \theta / 2} x\right),
$$

where $h_{k}$ denote (normalized) Hermite functions. The eigenfunctions of the adjoint $H^{*}$ are obtained by complex conjugation, and it is easy to verify the biorthonormal relation

$$
\left\langle h_{k}\left(e^{i \theta / 2} x\right), h_{l}\left(e^{-i \theta / 2} x\right)\right\rangle=\delta_{k l} .
$$

One may show that the eigenfunctions are complete in $L^{2}(\mathbb{R})$, the corresponding eigenvalues are algebraically simple, and there are no other points in the spectrum, cf. 22]. Consequently, the spectral projections $P_{k}$ of $H$ can be written as

$$
P_{k}=h_{k}\left(e^{i \theta / 2} x\right)\left\langle h_{k}\left(e^{-i \theta / 2} x\right), \cdot\right\rangle .
$$

The Cauchy-Schwarz inequality, the biorthonormal relation (89), and symmetries of the Hermite functions can be used to show that

$$
\left\|P_{k}\right\|=\left\|h_{k}\left(e^{i \theta / 2} x\right)\right\|\left\|h_{k}\left(e^{-i \theta / 2} x\right)\right\|=\left\|h_{k}\left(e^{i \theta / 2} x\right)\right\|^{2} .
$$

The resulting norms can be calculated explicitly. As pointed out by F. Bagarello in [13, the formula [59, Eq. 2.20.16.2, p. 502]

$$
\begin{aligned}
& \int_{0}^{\infty} e^{-a x^{2}} H_{k}(b x) H_{k}(c x) \mathrm{d} x \\
& \quad=\frac{2^{k-1} k ! \sqrt{\pi}}{a^{(k+1) / 2}}\left(b^{2}+c^{2}-a\right)^{k / 2} \mathcal{P}_{k}\left(\frac{b c}{\sqrt{a\left(b^{2}+c^{2}-a\right)}}\right),
\end{aligned}
$$

which is valid if $\operatorname{Re} a>0$ and where $\mathcal{P}_{k}$ are the Legendre polynomials, yields

$$
\left\|P_{k}\right\|=\frac{1}{(\cos \theta)^{1 / 2}} \mathcal{P}_{k}\left(\frac{1}{\cos \theta}\right) .
$$

The final result comes from the asymptotic behaviour of $\mathcal{P}_{k}(x), c f$. the LaplaceHeine formula [76, Thm. 8.21.1] or its generalization [76, Thm. 8.21.2] which provides further terms:

$$
\left\|P_{k}\right\|=\frac{1}{\sqrt{2 \pi k|\sin \theta|}}\left(\frac{1+|\sin \theta|}{\cos \theta}\right)^{k+1 / 2}(1+o(1)) .
$$

We note that this exponential factor agrees with (37) and with [27, 38, following a simple computation in [79, Ex. 3.6]. 


\section{Acknowledgment}

The work has been partially supported by the project RVO61389005, the GACR grant No. 14-06818S and the MOBILITY project 7AMB12FR020. P.S. acknowledges the SCIEX Programme, the work has been conducted within the SCIEX-NMS Fellowship, project 11.263, and thanks F. Bagarello for showing and discussing his results 13 during the research visit in Palermo in October 2013. J.V. is grateful for the support of ANR NOSEVOL (Project number: ANR 2011 BS01019 01).

\section{References}

[1] Recall that "self-adjointness" of a densely defined operator $H$, i.e. $H=H^{*}$, is a much stronger property than being just "Hermitian" or "symmetric", i.e. $H \subset H^{*}$. On the other hand, the terms "non-Hermitian" and "non-self-adjoint" can be read as synonyms in the present paper, since we are concerned with a "fundamental non-self-adjointness" caused for instance by complex coefficients of differential operators; that is, we are not interested in closed symmetric non-self-adjoint operators here.

[2] Bounded and boundedly invertible $\Omega$ means $\Omega, \Omega^{-1} \in \mathscr{B}(\mathcal{H})$, where $\mathscr{B}(\mathcal{H})$ denotes the space of bounded operators on $\mathcal{H}$ to $\mathcal{H}$.

[3] R. A. Adams. Sobolev Spaces. Academic Press, New York, 1975.

[4] J. Adduci and B. Mityagin. Eigensystem of an $L^{2}$-perturbed harmonic oscillator is an unconditional basis. Central European Journal of Mathematics, 10:569-589, 2012.

[5] J. Adduci and B. Mityagin. Root system of a perturbation of a selfadjoint operator with discrete spectrum. Integral Equations Operator Theory, 73:153-175, 2012.

[6] Z. Ahmed. Pseudo-Hermiticity of Hamiltonians under gauge-like transformation: real spectrum of non-Hermitian Hamiltonians. Physics Letters A, 294:287-291, 2002.

[7] S. Albeverio, U. Guenther, and S. Kuzhel. J-self-adjoint operators with C-symmetries: extension theory approach. Journal of Physics A: Mathematical and Theoretical, 42:105205, 2009.

[8] A. Aleman and J. Viola. On weak and strong solution operators for evolution equations coming from quadratic operators. arXiv:1409.1262, September 2014.

[9] A. Aleman and J. Viola. Singular-value decomposition of solution operators to model evolution equations. arXiv:1409.1255, September 2014.

[10] Y. Almog. The stability of the normal state of superconductors in the presence of electric currents. SIAM Journal on Mathematical Analysis, 40:824-850, 2008.

[11] Y. Almog, B. Helffer, and X.-B. Pan. Superconductivity near the Normal State in A HalfPlane under the Action of A Perpendicular Electric Current and an Induced Magnetic Field, Part II: The Large Conductivity Limit. SIAM Journal on Mathematical Analysis, 44:3671-3733, 2012.

[12] Y. Almog, B. Helffer, and X.-B. Pan. Superconductivity near the Normal State in a HalfPlane under the Action of a Perpendicular Electric Currents and an Induced Magnetic Field. Transactions of the American Mathematical Society, 365:1183-1217, 2013.

[13] F. Bagarello. Examples of pseudo-bosons in quantum mechanics. Physics Letters A, 374:3823-3827, 2010.

[14] C. M. Bender. Making sense of non-Hermitian Hamiltonians. Reports on Progress in Physics, 70:947-1018, 2007.

[15] C. M. Bender and P. N. Boettcher. Real spectra in non-Hermitian Hamiltonians having PT symmetry. Physical Review Letters, 80:5243-5246, 1998.

[16] C. M. Bender, D. C. Brody, and H. F. Jones. Complex extension of quantum mechanics. Physical Review Letters, 89:270401, 2002.

[17] J. Blank, P. Exner, and M. Havlíček. Hilbert space operators in quantum physics. Springer, 2008. 
[18] W. Bordeaux Montrieux. Estimation de résolvante et construction de quasimode près du bord du pseudospectre. arXiv:1301.3102 [math.SP] (2013).

[19] D. Borisov and D. Krejčiřík. PT-symmetric waveguides. Integral Equations and Operator Theory, 62:489-515, 2008.

[20] D. Borisov and D. Krejčiřík. The effective Hamiltonian for thin layers with non-Hermitian Robin-type boundary conditions. Asympt. Anal., 76:49-59, 2012.

[21] E. Caliceti, S. Graffi, M. Hitrik, and J. Sjöstrand. Quadratic P $\mathcal{P}$-symmetric operators with real spectrum and similarity to self-adjoint operators. Journal of Physics A: Mathematical and Theoretical, 45:444007, 2012.

[22] E. B. Davies. Pseudo-spectra, the harmonic oscillator and complex resonances. Proceedings of the Royal Society A, 455:585-599, 1999.

[23] E. B. Davies. Semi-classical states for non-self-adjoint Schrödinger operators. Communications in Mathematical Physics, 200:35-41, 1999.

[24] E. B. Davies. Wild spectral behaviour of anharmonic oscillators. Bulletin London Mathematical Society, 32:432-438, 2000.

[25] E. B. Davies. Non-self-adjoint differential operators. Bulletin London Mathematical Society, 34:513-532, 2002.

[26] E. B. Davies. Linear operators and their spectra. Cambridge University Press, 2007.

[27] E. B. Davies and A. B. J. Kuijlaars. Spectral asymptotics of the non-self-adjoint harmonic oscillator. Journal of the London Mathematical Society, 70:420-426., 2004.

[28] E. B. Davies and M. Marletta. Private communication, Edinburgh, 2013.

[29] N. Dencker, J. Sjöstrand, and M. Zworski. Pseudospectra of Semiclassical (Pseudo-) Differential Operators. Communications on Pure and Applied Mathematics, 57:384-415, 2004.

[30] J. Dieudonné. Quasi-Hermitian operators. Proceedings of the International Symposium on Linear Spaces, pages 115-123, 1961.

[31] M. Dimassi and J. Sjöstrand. Spectral Asymptotics in the Semi-Classical Limit. London Mathematical Society, 1999.

[32] P. Dorey, C. Dunning, and R. Tateo. Spectral equivalences, Bethe ansatz equations, and reality properties in $\mathcal{P} \mathcal{T}$-symmetric quantum mechanics. Journal of Physics A: Mathematical and General, 34:5679-5704, 2001.

[33] D. E. Edmunds and W. D. Evans. Spectral theory and differential operators. Oxford University Press, 1987.

[34] M. E. Fisher. Yang-Lee edge singularity and $\varphi^{3}$ field theory. Physical Review Letters, 40:1610-1613, 1978.

[35] I. Giordanelli and G. M. Graf. The Real Spectrum of the Imaginary Cubic Oscillator: An Expository Proof. Ann. Henri Poincaré, pages 1-14, 2014.

[36] E.-M. Graefe, H. J. Korsch, A. Rush, and R. Schubert. Classical and quantum dynamics in the (non-Hermitian) Swanson oscillator. Journal of Physics A: Mathematical and Theoretical, 48:055301, 2015.

[37] S. Hassi and S. Kuzhel. On $J$-self-adjoint operators with stable $C$-symmetry. Proceedings of the Royal Society of Edinburgh A, 143:141-167, 2013.

[38] R. Henry. Spectral instability for even non-selfadjoint anharmonic oscillators. Journal of Spectral Theory, 4:349-364, 2014.

[39] R. Henry. Spectral Projections of the Complex Cubic Oscillator. Annales Henri Poincaré, 15:2025-2043, 2014

[40] I. W. Herbst. Dilation analyticity in constant electric field. I. The two body problem. Communications in Mathematical Physics, 64(3):279-298, 1979.

[41] M. Hitrik, J. Sjöstrand, and J. Viola. Resolvent estimates for elliptic quadratic differential operators. Analysis \& PDE, 6:181-196, 2013.

[42] L. Hörmander. Symplectic classification of quadratic forms, and general Mehler formulas. Mathematische Zeitschrift, 219(3):413-449, 1995. 
[43] L. Hörmander. The analysis of linear partial differential operators. III. Classics in Mathematics. Springer, Berlin, 2007. Pseudo-differential operators, Reprint of the 1994 edition.

[44] T. Kato. Perturbation theory for linear operators. Springer-Verlag, Berlin, 1995.

[45] D. Krejčiř́ík, P. Siegl, and J. Železný. On the similarity of Sturm-Liouville operators with non-Hermitian boundary conditions to self-adjoint and normal operators. Complex Analysis and Operator Theory, 8:255-281, 2014.

[46] D. Krejčiřík. Calculation of the metric in the Hilbert space of a $\mathcal{P} \mathcal{T}$-symmetric model via the spectral theorem. Journal of Physics A: Mathematical and Theoretical, 41:244012, 2008.

[47] D. Krejčiřík, H. Bíla, and M. Znojil. Closed formula for the metric in the Hilbert space of a $\mathcal{P T}$-symmetric model. Journal of Physics A: Mathematical and General, 39:1014310153, 2006.

[48] D. Krejčiřík and Siegl. PT-symmetric models in curved manifolds. Journal of Physics A: Mathematical and Theoretical, 43:485204, 2010.

[49] D. Krejčiřík and M. Tater. Non-Hermitian spectral effects in a $\mathcal{P} \mathcal{T}$-symmetric waveguide. J. Phys. A: Math. Theor., 41:244013, 2008.

[50] B. Mityagin and P. Siegl. Root system of singular perturbations of the harmonic oscillator type operators. Letters in Mathematical Physics, 2015 (to appear).

[51] B. Mityagin, P. Siegl, and J. Viola. Differential operators admitting various rates of spectral projection growth. arXiv:1309.3751, 2013.

[52] A. Mostafazadeh. Pseudo-Hermiticity versus PT symmetry: The necessary condition for the reality of the spectrum of a non-Hermitian Hamiltonian. Journal of Mathematical Physics, 43:205-214, 2002.

[53] A. Mostafazadeh. Pseudo-Hermiticity versus PT symmetry: II. A complete characterization of non-Hermitian Hamiltonians with a real spectrum. Journal of Mathematical Physics, 43:2814-2816, 2002.

[54] A. Mostafazadeh. Pseudo-Hermiticity versus PT symmetry: III. Equivalence of pseudoHermiticity and the presence of antilinear symmetries. Journal of Mathematical Physics, 43:3944-3951, 2002.

[55] A. Mostafazadeh. Pseudo-Hermitian Representation of Quantum Mechanics. International Journal of Geometric Methods in Modern Physics, 7:1191-1306, 2010.

[56] K. Pravda-Starov. A complete study of the pseudo-spectrum for the rotated harmonic oscillator. Journal of the London Mathematical Society, 73:745-761, 2006.

[57] K. Pravda-Starov. Boundary pseudospectral behaviour for semiclassical operators in one dimension. International Mathematics Research Notices. IMRN, pages Art. ID rnm 029, $31,2007$.

[58] K. Pravda-Starov. On the pseudospectrum of elliptic quadratic differential operators. Duke Mathematical Journal, 145:249-279, 2008.

[59] A. P. Prudnikov, Y. A. Brychkov, and O. I. Marichev. Integrals and Series, volume 2. Gordon and Breach Science Publishers, Amsterdam, 1986. [Special functions].

[60] S. C. Reddy and L. N. Trefethen. Pseudospectra of the convection-diffusion operator. SIAM Journal on Applied Mathematics, 54:1634-1649,, 1994.

[61] M. Reed and B. Simon. Methods of Modern Mathematical Physics, I. Functional Analysis. Academic Press, New York, 1972.

[62] M. Reed and B. Simon. Methods of Modern Mathematical Physics, IV. Analysis of Operators. Academic Press, New York, 1978.

[63] L. Reichel and L. N. Trefethen. Eigenvalues and pseudo-eigenvalues of Toeplitz matrices. Linear Algebra and its Applications, 162/164:153-185, 1992.

[64] S. Roch and B. Silberman. $C^{*}$-algebra techniques in numerical analysis. Journal of Operator Theory, 35:241-280, 1996.

[65] M. Schechter. Operator methods in quantum mechanics. Elsevier, 1981.

[66] F. G. Scholtz, H. B. Geyer, and F. J. W. Hahne. Quasi-Hermitian operators in quantum mechanics and the variational principle. Annals of Physics, 213:74-101, 1992. 
[67] E. Shargorodsky. On the definition of pseudospectra. Bulletin of the London Mathematical Society, 41:524-534, 2009.

[68] E. Shargorodsky. Pseudospectra of semigroup generators. Bulletin of the London Mathematical Society, 42:1031-1034, 2010.

[69] K. C. Shin. On the reality of the eigenvalues for a class of $\mathcal{P} \mathcal{T}$-symmetric oscillators. Communications in Mathematical Physics, 229:543-564, 2002.

[70] P. Siegl. PT-Symmetric Square Well-Perturbations and the Existence of Metric Operator. International Journal of Theoretical Physics, 50:991-996, 2011.

[71] P. Siegl and D. Krejčiřík. On the metric operator for the imaginary cubic oscillator. Physical Review D, 86:121702(R), 2012.

[72] B. Simon. The bound state of weakly coupled Schrödinger operators in one and two dimensions. Annales de Physique, 97:279-288, 1976.

[73] J. Sjöstrand. Parametrices for pseudodifferential operators with multiple characteristics. Arkiv för Matematik, 12:85-130, 1974.

[74] J. Sjöstrand. Singularités analytiques microlocales. In Astérisque, 95, volume 95 of Astérisque, pages 1-166. Soc. Math. France, Paris, 1982.

[75] M. S. Swanson. Transition elements for a non-Hermitian quadratic Hamiltonian. Journal of Mathematical Physics, 45:585-601, 2004.

[76] G. Szegö. Orthogonal polynomials. American Mathematical Society, Providence, R.I., 1959.

[77] L. N. Trefethen and M. Embree. Spectra and pseudospectra. Princeton University Press, 2005.

[78] L. N. Trefethen et al. Chebfun Version 4.2. The Chebfun Development Team, 2011. http://www. chebfun.org/.

[79] J. Viola. Spectral projections and resolvent bounds for partially elliptic quadratic differential operators. Journal of Pseudo-Differential Operators and Applications, 4:145-221, 2013.

[80] M. Znojil. PT-symmetric square well. Physics Letters A, 285:7-10, 2001.

[81] M. Zworski. A remark on a paper of E. B. Davies. Proceedings of the American Mathematical Society, 129:2955-2957, 2001.

[82] M. Zworski. Semiclassical analysis, volume 138 of Graduate Studies in Mathematics. American Mathematical Society, Providence, RI, 2012. 